\title{
Characterization of Progenitor Domains in the Developing Mouse Thalamus
}

\author{
TOU YIA VUE, ${ }^{1-3}$ JOSHUA AAKER, ${ }^{1,2}$ AYA TANIGUCHI, ${ }^{1,2}$ \\ CHRISTINA KAZEMZADEH, ${ }^{1,2}$ JENNIFER M. SKIDMORE, ${ }^{4}$ DONNA M. MARTIN, ${ }^{4}$ \\ JAMES F. MARTIN, ${ }^{5}$ MATHIAS TREIER, ${ }^{6}$ AND YASUSHI NAKAGAWA ${ }^{1-3 *}$ \\ ${ }^{1}$ Department of Neuroscience, University of Minnesota, Minneapolis, Minnesota 55455 \\ ${ }^{2}$ Stem Cell Institute, University of Minnesota, Minneapolis, Minnesota 55455 \\ ${ }^{3}$ Graduate Program in Neuroscience, University of Minnesota, Minneapolis, Minnesota 55455 \\ ${ }^{4}$ Departments of Pediatrics and Human Genetics, University of Michigan, \\ Ann Arbor, Michigan 48109 \\ ${ }^{5}$ Institute of Biosciences and Technology, Texas A\&M Systems Health Science Center, \\ College Station, Texas \\ ${ }^{6}$ Developmental Biology Unit, EMBL, Heidelberg, Germany
}

\begin{abstract}
To understand the molecular basis of the specification of thalamic nuclei, we analyzed the expression patterns of various transcription factors and defined progenitor cell populations in the embryonic mouse thalamus. We show that the basic helix-loop-helix (bHLH) transcription factor Olig3 is expressed in the entire thalamic ventricular zone and the zona limitans intrathalamica (ZLI). Next, we define two distinct progenitor domains within the thalamus, which we name pTH-R and pTH-C, located caudal to the ZLI. pTH-R is immediately caudal to the ZLI and expresses Nkx2.2, Mash1, and Olig3. pTH-C is caudal to pTH-R and expresses Ngn1, Ngn2, and Olig3. Short-term lineage analysis of Olig3-, Mash1-, Ngn1-, and Ngn2-expressing progenitor cells as well as tracing the Pitx2 cell lineage suggests that pTH-C is the only major source of thalamic nuclei containing neurons that project to the cerebral cortex, whereas pTH-R and ZLI are likely to produce distinct postmitotic populations outside of the cortex-projecting part of the thalamus. To determine if pTH-C is composed of subdomains, we characterized expression of the homeodomain protein Dbx1 and the bHLH protein Olig2. We show that Dbx1 is expressed in caudodorsal-high to rostroventral-low gradient within pTH-C. Analysis of heterozygous $D b x 1^{\text {nlslac } Z}$ knockin mice demonstrated that Dbx1-expressing progenitors preferentially give rise to caudodorsal thalamic nuclei. Olig2 is expressed in an opposite gradient within pTH-C to that of Dbx1. These results establish the molecular heterogeneity within the progenitor cells of the thalamus, and suggest that such heterogeneity contributes to the specification of thalamic nuclei. J. Comp. Neurol. 505:73-91, 2007. ๑ 2007 Wiley-Liss, Inc.
\end{abstract}

Indexing terms: patterning; thalamus; transcription factors; Sonic hedgehog; bHLH factors; lineage tracing

Sensory inputs from the periphery are vital for all animal species to adapt and survive in the environment. The thalamus is composed of dozens of nuclei, some of which are major relay centers of sensory inputs into the neocortex (Jones, 2007). In addition, it has been proposed that many of the thalamic nuclei play roles in cortico-cortical communications and motor control (Sherman and Guillery, 2005), as well as in cognitive events through thalamocortical synchrony (Jones, 2001). Thus, understanding the mechanisms that control the specification and differentiation of thalamic nuclei is crucial for understanding how animal behavior develops. However, very
Grant sponsor: GENSAT Project supported by NINDS; Grant number: N01NS02331 (to Rockefeller University); Grant sponsor: National Institutes of Health (NIH); Grant number: R01DE16329 (to J.M.M.).

*Correspondence to: Yasushi Nakagawa, Department of Neuroscience, University of Minnesota, Minneapolis, MN 55455.

E-mail: nakagawa@umn.edu

Received 7 December 2006; Revised 8 June 2007; Accepted 29 June 2007 DOI 10.1002/cne.21467

Published online in Wiley InterScience (www.interscience.wiley.com). 
little is known about how individual thalamic nuclei are specified during embryogenesis. Early in development, the thalamic primordium appears in the alar plate of the caudal diencephalon (Puelles and Rubenstein, 1993), flanked rostrally by zona limitans intrathalamica (ZLI), a band of cells that express a signaling molecule, Sonic hedgehog (Shh; Echelard et al., 1993). Rostral to the ZLI is the prethalamus, and the caudal boundary of the thalamus separates the thalamus from the pretectum.

Thus far, studies of the development of the thalamus have mainly been pursued from two perspectives. One series of studies primarily focused on the roles of signaling molecules such as Shh, Wnts, and BMPs in the global patterning of the embryonic thalamus and its adjacent regions (Braun et al., 2003; Echevarria et al., 2003; Kiecker and Lumsden, 2004; Zhou et al., 2004; Lim et al., 2005; Lim and Golden, 2007). Using chick embryos, some of these studies have proposed that Shh produced by the ZLI plays a critical role. For example, ectopic expression of Shh induces the expression of thalamic markers such as Gbx2 and Sox14 in tissues caudal to the ZLI.

The second series of studies focused on identifying genes and proteins that are differentially expressed in distinct postmitotic thalamic nuclei. Expression patterns of a number of transcription factors have been mapped to different thalamic nuclei in the chick, mouse, and monkey (Miyashita-Lin et al., 1999; Nakagawa and O'Leary, 2001, 2003; Lim and Golden, 2002; Martinez-de-la-Torre et al., 2002; Hashimoto-Torii et al., 2003; Seibt et al., 2003; Jones and Rubenstein, 2004). These genes include Ngn2, Gbx2, Lhx2, Lhx9, Sox2, Sox14, Id2, Lef1, RORa, and $R O R b$. The roles of Ngn2 and Gbx2 in postmitotic thalamic cells have been reported (Miyashita-Lin et al., 1999; Seibt et al., 2003), but how these factors may control the specification of nuclei is still unknown. In addition to the above studies, detailed immunohistochemical examination of cadherin expression in the chick embryonic thalamus has been performed (Redies et al., 2000; Yoon et al., 2000). In those articles the authors proposed that the thalamic mantle zone is divided into secondary subdivisions, which are dorsal, intermediate, and ventral tiers as well as the anteroventral subregion. In particular, they noticed a stream of cadherin-6B-positive cells in the rostralmost part of the thalamus that appeared to migrate from the ventricular zone to form the anteroventral subregion (Yoon et al., 2000). However, the exact lineage relationships between dividing progenitor cells and their postmitotic progeny have remained elusive. Moreover, the extent to which specific postmitotic neuronal populations adopt transient or temporary fates during terminal differentiation is not known.

Recently, the use of genetic cell marking methods has enabled tracing the fates of progenitor cells expressing a particular gene, even after the progenitor cells lose the expression of the gene upon differentiation into neurons. One remarkable example is the early embryonic spinal cord, where the ventricular zone is dorsoventrally divided into multiple progenitor domains with distinct sets of transcription factor expression. Numerous studies have established lineage relationships between each of these progenitor domains and distinct classes of postmitotic interneurons and motor neurons, as well as the roles of differentially expressed transcription factors (Jessell, 2000; Helms and Johnson, 2003; Zhuang and Sockanathan, 2006; Briscoe and Novitch, 2007).
In contrast, it is not clearly understood if the thalamic ventricular zone contains such distinct progenitor domains and, if so, how these domains contribute to the formation of specific nuclei. Without such knowledge it is impossible to fully understand the molecular mechanisms controlling the specification of thalamic nuclei. By analyzing the expression of various transcription factors, we tested if these regulatory genes are differentially expressed in the ventricular zone of the thalamus, which we defined by the incorporation of an S-phase maker, bromodeoxyuridine (BrdU), as well as the expression of regional markers that define the rostral-caudal and dorsalventral extent of the thalamus. We found that Olig2, Olig3, Ngn1, Ngn2, Mash1, Nkx2.2, and Dbx1 are all expressed in the thalamic ventricular zone, and that they distinguish positionally different progenitor populations. We then performed short-term lineage tracing analyses of progenitors expressing Olig3, Ngn1, Ngn2, Mash1, or Dbx1, as well as the Cre-recombinase mediated permanent lineage tracing of cells expressing Pitx2, and found that each of these progenitors produces distinct postmitotic cell populations.

\section{MATERIALS AND METHODS}

\section{Animals}

Care and experimentation on mice were done in accordance with the Institutional Animal Care and Use Committee of the University of Minnesota. Gene expression patterns were analyzed using embryos of timed pregnant HSD/CD1/ICR mice (Charles River, Wilmington, MA, or Harlan, Indianapolis, IN). Lineage tracing experiments were done using heterozygous embryos of Olig3 $3^{G F P-I R E S n l s l a c Z}$ (Muller et al., 2005), Dbx $1^{\text {nlslac } Z}$ (Pierani et al., 2001), Ngn2 ${ }^{\text {KIEGFP }}$ (Seibt et al., 2003), Mash $1^{\text {EGFP }}$ (Gong et al., 2003) and Ngn $1^{\text {EGFP }}$ (Gong et al., 2003) mice. Pitx $2^{\text {crel+ }}$ mice (Liu, 2003) were mated to transgenic mice containing a Cre conditional nuclear localized $l a c Z$ reporter under the control of the chicken $\beta$-actin promoter ( $N$-lacZ) (Zinyk et al., 1998). Noon of the day on which the vaginal plug was found was counted as embryonic (E) day 0.5 . In addition to the plug date, we used gross anatomical features to accurately stage embryos younger than E14.5 (Kaufman, 1992). Olig3 $3^{\text {GFP-IRESnlslacZ }}$, Dbx $1^{\text {nlslac }}$, and Pitx $2^{\text {cre }}$ mice were maintained as heterozygotes in a C57BL/6J background, whereas Mash $1^{E G F P}$ and $N g n 1^{E G F P}$ BAC transgenic mice were maintained as heterozygotes in an FVB/N background. Expression patterns of the same gene at the same developmental stage did not differ between embryos from different strain backgrounds (e.g., C57B6/J vs. HSD/CD1/ICR). Genotyping of embryos was performed by polymerase chain reaction (PCR) using the following oligonucleotide primers: for Olig3 ${ }^{G F P-I R E S n l s l a c Z /+}$, Mash $1^{E G F P /+}$, and $N g n 1^{E G F P /+}$, 5'-AAT TCC TGC CTA AAG GCT CCT CAA C-3', and 5'-GTG CAG ATG AAC TTC AGG GTC AGC T-3', for Dbx $1^{\text {nlslac } Z 1+}, 5^{\prime}$-GGA GAG GCT ATT CGG CTA TGA-3' and 5'-CTT 'TCT CGG CAG GAG CAA GGT-3', for Pitx2Cre, 5'-GGT GGG GTG GGG GTG TCT GTA AAA-3' (for wildtype allele), 5'-GCT AGG CGC GAA GGT TCT CCA GTG-3' (for both wildtype and mutant alleles), and 5'-AGA TAT GGC CCG CGC TGG AGT TTC-3' (for mutant allele). 


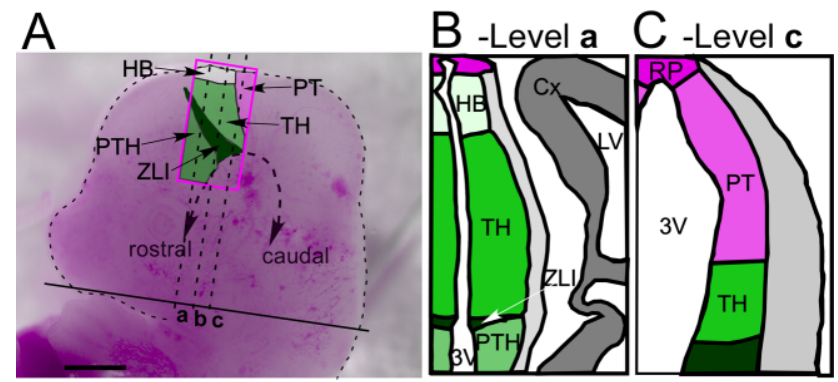

Fig. 1. Frontal sections used in this study. A: A whole-mount image of E12.5 brain showing the frontal sections used in this study. The embryo is facing toward the left. The rostrocaudal axis of the brain (dotted arrow) curves dramatically from the midbrain into the forebrain, making it impossible to cut sections that are constantly coronal. Dotted lines a, b, and $\mathbf{c}$ show typical frontal sections. They are roughly perpendicular to the base of the forebrain (solid black line) but have different angles against the rostrocaudal axis; section a is close to horizontal, while section $\mathbf{c}$ is closer to coronal. Among these three sections, $\mathbf{a}$ is the most dorsal and rostral, and $\mathbf{c}$ is the most ventral and caudal. B,C: How typical type a and type cections look. In both sections, caudodorsal is to the top and rostroventral is to the bottom. Type a sections (B) include the habenula (HB), the thalamus (TH), the zona limitans intrathalamica (ZLI), and the prethalamus (PTH) from the top to the bottom, and type c sections (C) include the pretectum (PT), the thalamus, and the ZLI. Cx, cortex; 3V, third ventricle; $L V$, lateral ventricle; $R P$, roof plate. Scale bar $=1 \mathrm{~mm}$ in A.

\section{Tissue preparation}

Pregnant female mice were intraperitoneally injected with BrdU (100 $\mu \mathrm{g} / \mathrm{g}$ body weight; Sigma, St. Lois, MO) an hour prior to sacrifice. Embryos were removed and dissected in $0.1 \mathrm{M}$ phosphate buffer, immersion-fixed in $4 \%$ paraformaldehyde in $0.1 \mathrm{M}$ phosphate buffer for 1 hour to overnight, submerged in 30\% sucrose, and frozen in OCT compound (EM Sciences, Hatfield, PA) on liquid nitrogen.

\section{Axial and anatomical nomenclature}

To maintain consistency in sectioning, we cut $20-\mu \mathrm{m}$ thick frontal sections. This is the same as the "coronal" sections that have been traditionally used for cutting rodent forebrain. This section plain is roughly perpendicular to the base of the forebrain, which is evident for older embryos when the brain is laid flat on its bottom. The "base" is actually formed by the anteriormost part of the brain, connecting the ventromedian hypothalamus, the preoptic area, and the septal nuclei (Puelles and Rubenstein, 2003; Puelles et al., 2004). As shown in Figure 1A, caudal is to the top and rostral is to the bottom in these sections. At the same time, because of the obliquity of the sections, dorsal is to the top and ventral is to the bottom. The alar plate of the caudal diencephalon contains three transverse domains: the pretectum (prosomere 1, most caudal), the habenula and the thalamus (prosomere 2 , the habenula is dorsal to the thalamus), and the prethalamus (prosomere 3, most rostral) (Puelles and Rubenstein, 2003). With our method, rostrodorsal sections (e.g., section a in Fig. 1A) contain (top) roof plate-habenula-thalamusZLI-prethalamus (bottom), and other, more caudoventral sections (e.g., section $\mathbf{c}$ in Fig. 1A) contain (top) roof platepretectum-thalamus-ZLI-basal plate (bottom). Some type c sections contain prethalamus below the ZLI. Type a and type c sections can be distinguished by morphology or by markers expressed in the pretectum but not in the habenula, such as Lhx1 and Mab21L1 (Fig. 6). Diagrams of typical sections at two different levels $(\mathbf{a}, \mathbf{c})$ are shown (Fig. 1B,C). Thalamic, prethalamic, and pretectal nuclei were identified based on previous studies (Nakagawa and O'Leary, 2001) and an atlas (Paxinos, 1994).

\section{Immunostaining}

Cryosections were collected on Superfrost Plus slides (Fisher, Pittsburgh, PA) and dried on a slide warmer for at least 30 minutes. Sections were then rinsed in phosphatebuffered saline (PBS), immersed in boiled $10 \mathrm{mM}$ citrate buffer $(\mathrm{pH} 6)$ for 5 minutes prior to blocking with $3 \%$ donkey serum / 0.3\% Triton X-100/PBS for 1 hour. Primary antibodies were then added at appropriate dilutions and incubation was performed overnight at $4^{\circ} \mathrm{C}$. On the second day, after washing in PBS, sections were incubated with fluorochrome-conjugated secondary antibodies (Cy2, Cy3, or Cy5, from Jackson ImmunoResearch, West Grove, PA), followed by DAPI counterstaining. After dehydration in ascending concentrations of ethanol and clearing in xylene, slides were mounted in DPX mounting medium (EM Sciences, Hatfield, PA). For staining with anti-BrdU antibody, sections were fixed before treatment with $2 \mathrm{~N}$ $\mathrm{HCl} / 0.1 \%$ Triton X-100 for 20 minutes. After $4-5$ washes in PBS, anti-BrdU antibody was added and subsequent detection was done as described above for other antibodies.

Immunofluorescence was analyzed using an E800 upright microscope (Nikon, Tokyo, Japan) with a Retiga EXi camera (Qimaging, Burnaby, BC, Canada) and Open Lab software (Improvision, Lexington, MA), or Axioskop2 microscope (Carl Zeiss, Thornwood, NY) with a laser scanning confocal unit (BioRad Radiance 2100, Hercules, CA). Double- or triple-labeling was compared by combining color channels in Photoshop CS2 (Adobe, San Jose, CA). Analysis of gene expression gradient was done using the Image $J$ software using the Plot Profile command (NIH, Bethesda, MD).

\section{Antibodies}

The primary antibodies used in this study are listed in Table 1. For all the embryos younger than E14.5 we collected sections of both the forebrain and the spinal cord on the same slides and performed the immunohistochemical analysis side by side. We detected no discrepancy between our results on the spinal cord and published data using the same antibodies.

\section{Western blot analysis}

Caudal diencephalon and midbrain were dissected from E12.5 Dbx $1^{\text {nlslacZ/nlslac } Z}, D b x 1^{\text {nlslac } Z /+}$, and wildtype embryos of the same litter. The sample from each embryo was individually sonicated for 30 seconds in RIPA lysis buffer. Protein lysate from each embryo was mixed with sample buffer and run on a 10\% Tris-Glycine Durmaide gel (Cambrex Bioscience, Walkersville, MD). The proteins were transferred to an Immobilon-P PVDF membrane (Millipore, Billerica, MA) and blocked with $3 \%$ skim milk in PBS with $0.1 \%$ Tween-20. Anti-Dbx1 antibody was used 1:100. The secondary goat antirabbit horseradish peroxidase labeled antibody was used at 1:5,000 (Pierce, Rockford, IL). The membrane was washed after each antibody incubation with $0.1 \%$ Tween-20 in PBS. The blot was developed in Pierce's SuperSignal West Femto kit as per 
TABLE 1. List of Primary Antibodies

\begin{tabular}{|c|c|c|c|c|c|}
\hline $\begin{array}{l}\text { Primary } \\
\text { antibody }\end{array}$ & Source & $\begin{array}{l}\text { Catalog no. } \\
\text { Lot no. }\end{array}$ & Immunogen & Dilution & Specificity \\
\hline Mouse anti-Olig3 & $\begin{array}{l}\text { R\&D Systems, } \\
\text { Minneapolis, MN }\end{array}$ & MAB2456 & $\begin{array}{l}\text { Recombinant human Olig3 (amino acid } \\
\text { 1-272) }\end{array}$ & $1: 500$ & $\begin{array}{l}\text { No crossreactivity to Olig1 or Olig2 on immunoblot. } \\
\text { Shows the same pattern as mRNA on in situ } \\
\text { hybridization }\end{array}$ \\
\hline Rabbit anti-Olig3 & Thomas Müller & - & $\begin{array}{l}\text { Recombinant mouse full-length Olig3 with } \\
\text { His tag }\end{array}$ & $1: 1,000$ & $\begin{array}{l}\text { Shows the same pattern as MAB2456 on double } \\
\text { immunofluorescence (Müller et al., 2005) }\end{array}$ \\
\hline Mouse anti-Ngn2 & $\begin{array}{l}\text { R\&D Systems, } \\
\text { David Anderson }\end{array}$ & $\begin{array}{l}\text { MAB3314, (clone } \\
\text { 7G4) and clone } \\
\text { 5C6 }\end{array}$ & $\begin{array}{l}\text { Recombinant mouse Ngn2 containing the } \\
\text { N-terminus of the HLH domain (aa 1- } \\
110 \text { ) }\end{array}$ & $1: 100$ & $\begin{array}{l}\text { Detects a single band on immunoblot (Ge et al., } \\
2006 \text { ). Staining on sections is blocked by Ngn2- } \\
\text { GST protein but not by Ngn1-GST protein (Lo et } \\
\text { al., 2002) }\end{array}$ \\
\hline Goat anti-Mash1 & R\&D Systems & AF2567 & $\begin{array}{l}\text { E. coli-derived recombinant mouse Mash1 } \\
\text { (aa } 74-173)\end{array}$ & $1: 500$ & $\begin{array}{l}\text { Shows the same staining pattern as mRNA on in } \\
\text { situ hybridization }\end{array}$ \\
\hline Rabbit anti-Ngn1 & Jane Johnson & - & $\begin{array}{l}\text { E. coli-derived recombinant Ngn1 } \\
\text { containing the N-terminus part (aa } 1- \\
\text { 106). }\end{array}$ & $1: 200$ & No staining in Ngn1 knockout brains \\
\hline Goat anti-Sox2 & R\&D Systems & AF2018 & $\begin{array}{l}\text { E. coli-derived recombinant human Sox } 2 \\
\text { (aa 135-317) }\end{array}$ & $1: 100$ & $\begin{array}{l}\text { Shows specific binding on immunoblots. Less than } \\
1 \% \text { crossreactivity to Sox } 17\end{array}$ \\
\hline Goat anti-Shh & R\&D System & AF445 & $\begin{array}{l}\text { E. coli-derived recombinant mouse } \mathrm{Shh} \\
\text { purified against the N-terminal (aa } 25- \\
\text { 198) peptide }\end{array}$ & $1: 100$ & $\begin{array}{l}\text { Shows on immunoblot less than } 5 \% \text { crossreactivity } \\
\text { with Dhh C-terminal peptide and less than } 1 \% \\
\text { crossreactivity against Shh N-terminal peptide. }\end{array}$ \\
\hline $\begin{array}{l}\text { Mouse anti- } \\
\quad \mathrm{Nkx} 2.2\end{array}$ & $\begin{array}{l}\text { Developmental } \\
\text { Studies } \\
\text { Hybridoma Bank } \\
\text { (DSHB), Iowa } \\
\text { City, IA }\end{array}$ & 74.5A5 & E. coli-derived recombinant chick $\mathrm{Nkx} 2.2$ & $1: 1,000$ & $\begin{array}{l}\text { Detects a single band on immunoblot (Sun et al., } \\
2003 \text { ) }\end{array}$ \\
\hline Mouse anti-Pax7 & DSHB & PAX7 & $\begin{array}{l}\text { E. coli-derived recombinant chick Pax } 7 \\
\text { (aa } 352-523)\end{array}$ & $1: 10$ & $\begin{array}{l}\text { Detects a single band on immunoblot (Seale et al., } \\
2004 \text { ) }\end{array}$ \\
\hline Rabbit anti-NPY & $\begin{array}{l}\text { ImmunoStar, } \\
\text { Hudson, WI }\end{array}$ & 22940 & $\begin{array}{l}\text { Synthetic porcine NPY conjugated to } \\
\text { methylated bovine serum albumin }\end{array}$ & $1: 200$ & $\begin{array}{l}\text { All staining is blocked by preabsorption of the } \\
\text { diluted antiserum with excess NPY peptide }\end{array}$ \\
\hline $\begin{array}{l}\text { Goat anti- } \beta \text { - } \\
\text { galactosidase }\end{array}$ & $\begin{array}{l}\text { Biogenesis, } \\
\text { Brentwood, NH }\end{array}$ & $4600-1409$ & Native $\beta$-galactosidase from $E$. coli & $1: 500$ & Jansen et al., 1995 \\
\hline $\begin{array}{l}\text { Rabbit anti- } \beta- \\
\text { galactosidase }\end{array}$ & $\begin{array}{l}\text { Cappel/MP, } \\
\quad \text { Malvern, PA }\end{array}$ & 55976 & Native $\beta$-galactosidase from $E$. coli & $1: 500$ & Shows specificity on immunoblots \\
\hline Rat anti-BrdU & $\begin{array}{l}\text { Serotec, Bicester, } \\
\text { UK }\end{array}$ & MCA2060 & & $1: 200$ & $\begin{array}{l}\text { Recognizes BrdU incorporated into single stranded } \\
\text { DNA }\end{array}$ \\
\hline Mouse anti-TuJ1 & $\begin{array}{l}\text { Covance, Denver, } \\
\text { PA }\end{array}$ & MMS-435P & Microtubules derived from rat brain. & $1: 500$ & $\begin{array}{l}\text { Highly reactive and specific to neuron-specific Class } \\
\text { III } \beta \text {-tubulin on immunoblots }\end{array}$ \\
\hline Rabbit anti-GFP & $\begin{array}{l}\text { Invitrogen, La } \\
\text { Jolla, CA }\end{array}$ & A11122 & GFP isolated from Aequorea victoria & $1: 500$ & \\
\hline Rabbit anti-Dbx1 & & - & $\begin{array}{l}\text { KLH-conjugated peptide } \\
\text { (CDEDEEGEEDEEITVS) Injection and } \\
\text { serum preparation was done by Global } \\
\text { Peptide. We purified it by using } \\
\text { SulfoLink affinity column (Pierce) }\end{array}$ & $1: 200$ & $\begin{array}{l}\text { Detects a single band on immunoblot. No staining } \\
\text { on sections from Dbx1 knockout brains (see Fig. } \\
6 \mathrm{~W} \text { ) }\end{array}$ \\
\hline Goat anti-Olig2 & R\&D Systems & AF2418 & $\begin{array}{l}\text { E. coli-derived recombinant human Olig2 } \\
\text { (amino acid 1-323) }\end{array}$ & $1: 100$ & $\begin{array}{l}\text { On immunoblot, there is } 10 \% \text { crossreactivity with } \\
\text { recombinant Olig3 and } 5 \% \text { with recombinant } \\
\text { Olig1 }\end{array}$ \\
\hline
\end{tabular}

the manufacturer's instructions and exposed to film for 5 seconds.

\section{In situ hybridization}

In situ hybridization was performed according to Tuttle et al. (1999) with minor modifications. Digoxigeninlabeled probes used were: Olig3 (mouse, from H. Takebayashi, National Institute of Physiological Sciences, Okazaki, Japan), Gbx2 (mouse full-length clone, from G. Chapman, University of Adelaide, Adelaide, Australia); Dbx1 (mouse, Invitrogen, La Jolla, CA, clone \#5718470); BHLHB4 (mouse, Invitrogen clone \#6465274), and Mab21L1 (mouse, from N. Takahashi, University of Tokyo, Tokyo, Japan).

\section{RESULTS}

To identify distinct progenitor cell populations in the thalamus and the molecular mechanisms that specify their fates, we began with a detailed immunohistochemical analysis of a variety of transcription factors that are expressed differentially in the embryonic diencephalon. Many of these transcription factors have been shown to play important roles in the specification and differentiation of various neuronal types in other parts of the nervous system, and are thus likely to play similar roles in the thalamus. We characterized these transcription factors at E10.5, E11.5, and E12.5, stages at which most of the thalamic neurons are being generated (Angevine, 1970; Vue, Kazemzadeh, and Nakagawa, unpubl. data). In this study we defined progenitor cells as those in the ventricular zone, most of which are in the cell cycle. With the exception of Pitx2, all the transcription factors we analyzed show colocalization with the S-phase marker BrdU.

The frontal sections we used in this study (see sections a-c in Fig. 1A) are conventionally called "coronal." Due to a curvature of the rostrocaudal axis at the level of the diencephalon (Fig. 1A, dotted arrow), different frontal sections have different angles relative to the rostrocaudal axis. For example, section a is close to horizontal, while section $\mathbf{c}$ is closer to coronal. We chose to cut in this way because one section can contain more than one of the three transverse domains of the diencephalon in the alar plate, which are the prethalamus, the thalamus, and the pretectum (Fig. 1A-C). In addition, this section plane makes it easy to compare our current study with a number of previous studies. We consider these sections somewhere between coronal and horizontal. This axial nomenclature is in agreement with the prosomeric model of the vertebrate forebrain (Puelles and Rubenstein, 1993, 2003; Puelles, $1995,2001 \mathrm{a}, \mathrm{b})$. In this nomenclature, a type a section is more rostral and more dorsal than a type cection (Fig. 
1A-C). In a given frontal section, caudal as well as dorsal is oriented to the top, and rostral as well as ventral is oriented to the bottom. This nomenclature allows us to avoid confusion regarding the relative locations of thalamic nuclei. For example, medial geniculate nucleus is formed close to the ZLI in the ventral part of the thalamus, and thus should be regarded as a nucleus located rostrally as well as ventrally. In a conventional nomenclature, it is found in "caudal" sections (type c sections rather than type a sections) and is often wrongly mentioned as a caudal nucleus.

Figure 9 shows sagittal sections to show the expression patterns of some of the genes we analyzed in Figures 2-8. At the level of the ZLI, dorsal is almost to the left, and rostral is almost to the bottom in these sections (see Fig. 9B for axes). Planes of sections for images in Figures 2-8 are roughly perpendicular to the horizontal lines of the images in Figure 9.

\section{bHLH transcription factor Olig3 defines the entire thalamic ventricular zone}

We first characterized Olig3, a member of the basic helix-loop-helix (bHLH) family of transcription factors. Takebayashi et al. (2002) reported that Olig3 is expressed in the ventricular zone of the thalamus, but the details of its expression patterns or its functions within the diencephalon have not been reported.

In this study we performed immunofluorescence analysis of Olig3 in parallel with other genes and proteins that are expressed specifically in the thalamus and/or in its two surrounding regions, the prethalamus and the pretectum (Fig. 2). In situ hybridization shows that the Olig3 mRNA is expressed in the same pattern as the Olig3 protein (compare Fig. 6B,G). We first compared the expression pattern of Olig3 with that of $G b \times 2$, a homeobox gene whose expression patterns in the developing thalamus have been characterized in detail for chicks, mice, and monkeys (Bulfone et al., 1993; Miyashita-Lin et al., 1999; Nakagawa and O'Leary, 2001; Martinez-de-la-Torre et al., 2002; Hashimoto-Torii et al., 2003; Jones and Rubenstein, 2004). In the embryonic mouse thalamus, Gbx2 is expressed in specific nuclei, including the ventral part of the medial geniculate nucleus ( $\mathrm{MGv})$, central lateral, central medial, mediodorsal, posterior, and lateral posterior nuclei (Nakagawa and O'Leary, 2001; Jones and Rubenstein, 2004). Importantly, within the diencephalon Gbx2 is not expressed in the prethalamus, the habenula, or the pretectum, which makes $G b x 2$ a useful marker for the thalamic mantle zone. Gbx2 is required for normal differentiation of many thalamic nuclei in the mouse, as well as for the projection of thalamocortical axons to the neocortex (Miyashita-Lin et al., 1999).

Immunofluorescence analysis at E11.5 showed that Olig3 is expressed in the thalamic ventricular zone (Fig. $2 \mathrm{~A})$. Comparison with in situ hybridization for $G b \times 2$ on an adjacent section demonstrates that Olig3 and Gbx2 share the caudal and dorsal boundaries of expression (Fig. 2A,B, arrow), whereas Olig3 expression extends further rostroventrally than Gbx2 (Fig. 2A,B, asterisk). At E10.5, when only a small number of neurons have differentiated, Olig3 expression is found preferentially in the rostral part of the thalamic ventricular zone, with an apparent rostroventral-to-caudodorsal gradient (Fig. 2C, inset). However, similar to E11.5, the caudal and dorsal boundaries of Olig3 coincide with those of $G b x 2$, and Olig3

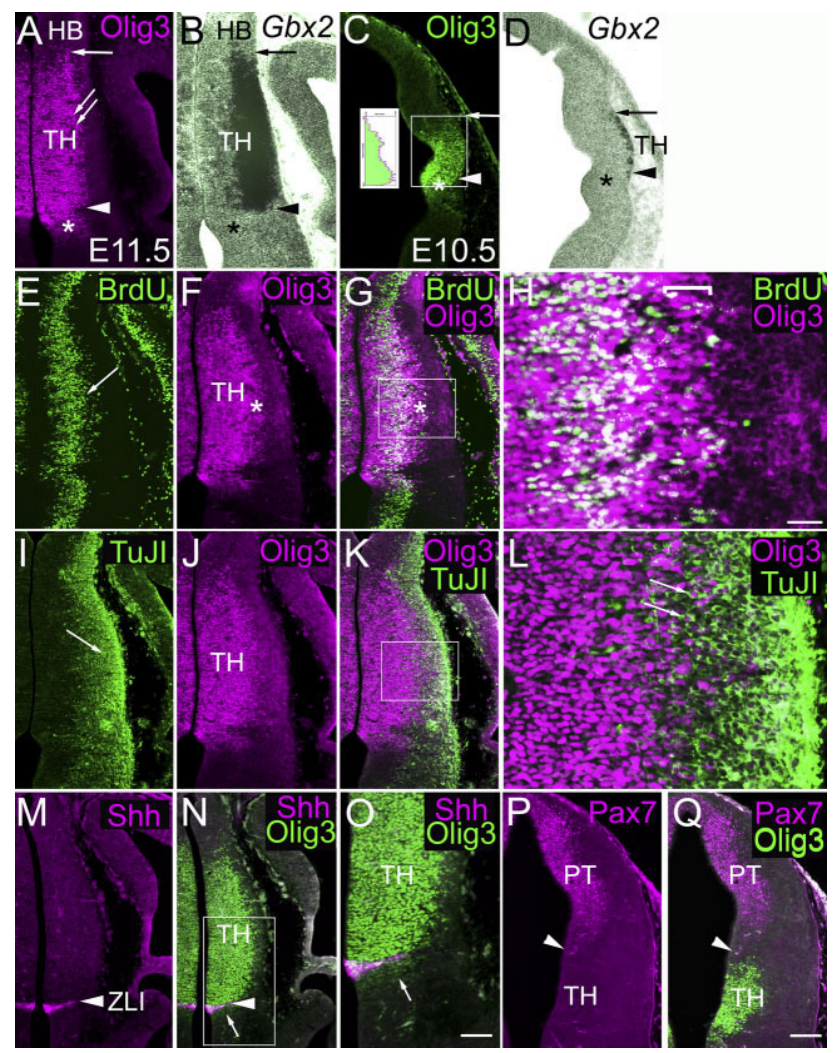

Fig. 2. Olig3 is expressed in the entire thalamic ventricular zone and the ZLI. Frontal sections from E11.5 (A,B,E-Q) or E10.5 (D,E) wildtype brains. Midline is to the left. A,B: Immunofluorescence for Olig3 (A) and in situ hybridization for $G b x 2$ (B) on adjacent sections at E11.5. The caudodorsal boundaries of Olig3 and Gbx2 expression match (arrow). The rostroventral boundary of Olig3 expression extends further than that of $G b x 2$ (arrowhead), into a separate domain (asterisk). Level of Olig3 expression within the thalamic ventricular zone is constant along the rostroventral-caudodorsal axis. C,D: The same comparison of Olig3 (C) and Gbx2 (D) at E10.5; the matching, caudodorsal boundary (arrow) and the nonmatching, rostroventral boundary (arrowhead and asterisk) are shown. At E10.5, Olig3 expression is higher rostroventrally and lower caudodorsally within the thalamus (C, inset for gradient of fluorescence intensity). E-H: Comparison of BrdU (E) and Olig3 (F) expression at E11.5 an hour after BrdU injection. Cells in S-phase of the cell cycle are BrdU-positive, and the lateral edge of high-density BrdU-positive cells indicates the lateral edge of the ventricular zone (E, arrow). $\mathrm{G}$ is a merged image of $\mathrm{E}$ and $\mathrm{F}$, and shows that Olig3 expression extends slightly further laterally from the lateral edge of the ventricular zone (asterisk). A higher-magnification image of the boxed area in $\mathrm{G}(\mathrm{H})$ shows that there is a band of Olig3-positive cells (bracket) outside of the ventricular zone that are BrdU-negative. I-L: Comparison of Olig3 and $\beta$-III-tubulin (detected byTuJ1 antibody) expression at E11.5. TuJ1positive cells are located in the mantle zone ( $\mathrm{I}$, arrow). $\mathrm{K}$ is a merged image of I and J. L is a high-magnification image of $K$, and shows that some Olig3-positive cells are in fact immunoreactive for TuJ1 (arrows), indicating that they are neurons. M-Q: Comparison of Olig3 expression with that of Shh $(\mathrm{M}-\mathrm{O})$ or Pax7 $(\mathrm{P}, \mathrm{Q})$ at E11.5. O is a high-magnification image of $\mathrm{N}$. The Shh-expressing ZLI is included in Olig3-expressing domain ( $\mathrm{N}$, arrowhead). In addition, Olig3 is expressed only at a very low level in the prethalamic ventricular zone, which is immediately rostral to the ZLI (N,O, arrow). Pax7, which is expressed in the pretectal ventricular zone, is not colocalized with Olig3 $(\mathrm{P}, \mathrm{Q})$. Arrowheads in $\mathrm{P}$ and $\mathrm{Q}$ show the boundary between the pretectum and the thalamus. Scale bars $=100 \mu \mathrm{m}$ for H,L,O; $200 \mu \mathrm{m}$ for other panels. 
expression extends further rostroventrally than $G b x 2$ (Fig. 2C,D, arrow and asterisk). Along the medial-lateral axis, Olig3 seems to overlap slightly with Gbx2 at the lateral margin of its expression domain (Fig. 2A, double arrows). To determine if Olig3-positive cells are proliferating, we pulse-labeled embryos for 1 hour prior to sacrifice and double-labeled with Olig3 and BrdU (Fig. 2E-H). A majority of Olig3-expressing cells are BrdU-positive, but there is a small band of cells just outside the ventricular zone (VZ) that is positive for Olig3 but mostly negative for BrdU (Fig. 2F,G, asterisk, and Fig. 2H, bracket). We further characterized cells in this region by staining with an antibody for neuron-specific $\beta$-III tubulin (TuJ1). Some of the TuJ1-positive cells in this region (Fig. 2L, arrows) also express Olig3, indicating that Olig3 is expressed not only in BrdU-positive progenitor cells but also in postmitotic neurons that are starting to express TuJ1.

To determine the rostral limit of Olig3 expression in more detail, we compared its expression with that of Shh, which marks the ZLI (Echelard et al., 1993). Double immunofluorescence shows that expression of Olig3 and Shh proteins heavily overlaps in the ZLI (Fig. 2M-O). Combined immunofluorescence analysis of Olig3 protein and in situ hybridization of Shh mRNA on the same section also confirmed this finding (data not shown). Expression of Olig3 further extends rostrally into the prethalamus, but the level of Olig3 expression in the prethalamus is far lower than that within the ZLI or the thalamus (Fig. $2 \mathrm{~N}, \mathrm{O}$, arrow). The same pattern was observed at E10.5 (data not shown). Sagittal sections also show that Olig3 and Shh overlap in the ZLI (Fig. 9A-D). To determine the caudal limit of Olig3 expression, we next compared expression of Olig3 and Pax7. The paired box transcription factor Pax7 is expressed in the pretectal ventricular zone (Matsunaga et al., 2001). We find that both at E10.5 (data not shown) and E11.5 (Fig. 2P,Q), Olig3 never overlaps with Pax7.

In summary, in the diencephalon Olig3 is expressed at a high level in the entire thalamic ventricular zone as well as in the ZLI, and at a much lower level in the caudal tip of the prethalamus.

\section{Expression patterns of Mash1 and Nkx2.2 are complementary to that of Neurogenin2 in the rostral thalamus}

To determine if there is molecular heterogeneity among progenitor cells within the Olig3-expressing thalamic ventricular zone, we next studied expression patterns of other transcription factors; we chose to analyze two other bHLH transcription factors, Mash1 and Neurogenin2 (Ngn2), and a homeodomain transcription factor Nkx2.2. Mash1 is expressed in a large domain within the ventricular zone of the prethalamus. In addition, Mash1 is expressed in a small patch of cells caudal to the prethalamus (Tuttle et al., 1999), but its precise location was unknown. Ngn2 mRNA is expressed broadly in the thalamus, both in the ventricular and mantle zones (Gradwohl et al., 1996; Fode et al., 2000; Nakagawa and O'Leary, 2001; Seibt et al., 2003). Analysis of knockout mice indicates that Ngn2 function in a partially redundant manner with its close member of the bHLH family, Ngn1, to restrict Mash1 expression to the prethalamus (Fode et al., 2000). Nkx2.2 is expressed in a domain straddling the ZLI (Price et al., 1992; Shimamura et al., 1995; Kitamura et al., 1997).
Based on the previous findings described above, we hypothesized that Ngn2, Mash1, and Nkx2.2 delineate the thalamic ventricular zone caudal to the ZLI. At E11.5 Mash1 is expressed in a large domain within the prethalamus (Fig. 3B, arrow). In addition, a small, separate domain caudal to the prethalamus also expresses Mash1 (Fig. 3B, arrowhead), which is similar to the previous finding on Mash1 mRNA (Tuttle et al., 1999). Triplestaining of Mash1, Olig3, and Shh shows that this small domain in the rostral thalamus expresses both Mash1 and Olig3 (Fig. 3D, arrow), whereas the ZLI, which expresses Shh, does not express Mash1 (Fig. 3E, arrowheads). The same pattern is observed at E10.5 (Fig. 3P-R). Thus, the small domain in the rostral thalamus with Mash1 expression is immediately caudal to the ZLI (Fig. 3E, asterisk), and is the most rostral progenitor domain in the thalamic ventricular zone; we call this domain pTH-R (R, rostral). Within pTH-R, some cells express both Mash1 and Olig3, but others express only one of the two proteins (Fig. 3D).

At both E10.5 and E11.5, Ngn2 is expressed broadly in a salt-and-pepper like pattern in the ventricular zone of the thalamus (Fig. 3G, double arrows). In addition, there is a small, separate band of expression more rostrally (Fig. $3 \mathrm{G}$, arrow). Double-staining with Shh confirms that this Ngn2-expressing domain is the ZLI (Fig. 3J, arrowheads). Double-staining with Ngn2 and Mash1 shows that they are expressed in mutually exclusive patterns, in which pTH-R expresses Mash1 but not Ngn2 (data not shown). We call the Ngn2-expressing domain of the thalamic ventricular zone pTH-C (C, caudal). Most of the Ngn2expressing cells within pTH-C and the ZLI also express Olig3 (Fig. 3I, arrows). Again, a similar pattern is already established at E10.5 (Fig. 3S-U).

Nkx2.2 is expressed on both sides of the ZLI, but not within the ZLI at E11.5 (Fig. 3L) and E10.5 (Fig. 3N). Within the thalamus, it is a small band of progenitor cells immediately caudal to the ZLI (Fig. 3L,O). This band of cells coincides with pTH-R, which expresses Mash1 (Fig. $3 \mathrm{E}, \mathrm{J}, \mathrm{O}$, asterisk). At E11.5, Nkx2.2 is also expressed in a strip of cells in the mantle zone of the diencephalon (Fig. 3L,M, double arrows).

In summary, the expression patterns of Ngn2, Mash1, and Nkx2.2 show that the thalamus contains at least two distinct progenitor domains, pTH-R and pTH-C; the pTH-R domain is adjacent to the ZLI and expresses Mash1 and Nkx2.2 but not Ngn2, and the caudally located pTH-C domain expresses Ngn2, but not Mash1 or Nkx2.2. Olig3 is expressed in both domains and also in the ZLI.

\section{Olig3-expressing progenitor cells contribute to all the cortex-projecting thalamic nuclei and other more rostral nuclei}

We next asked if progenitor cells of the pTH-R and pTH-C domains contribute to distinct nuclei of the postmitotic thalamus. First, we analyzed Olig3 GFP-IRESnlslac knockin mice (Muller et al., 2005). In these mice the coding region of Olig3 gene is replaced by both $\beta$-galactosidase $(\beta$-gal) and enhanced green fluorescent protein (EGFP). Thus, mice heterozygous for this allele are expected to produce $\beta$-gal and EGFP proteins in cells expressing endogenous Olig3. Although there is only one allele of Olig3 in these mice, we detect no changes in the expression of regional markers (data not shown). Because of their stability, $\beta$-gal and EGFP proteins remain detectable after 
E11.5
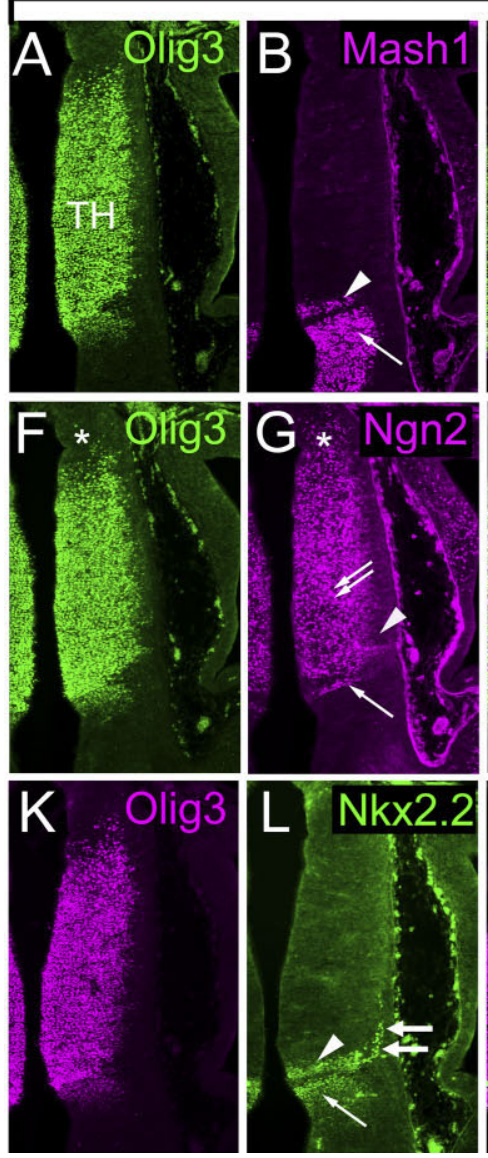
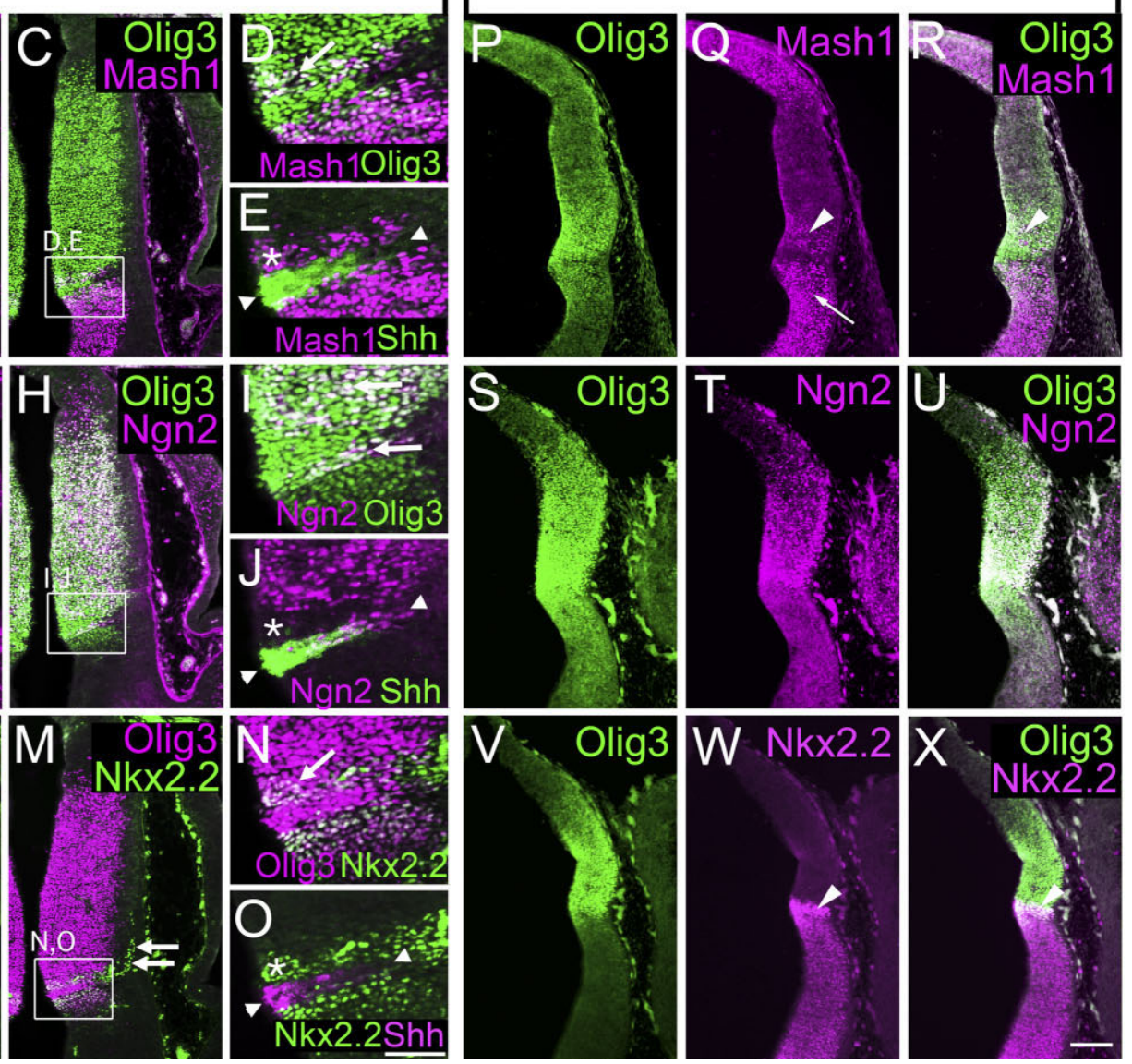

Fig. 3. Olig3-expressing thalamic ventricular zone is divided into two distinct domains. Immunofluorescence of frontal sections at E11.5 (A-O) and E10.5 (P-X) wildtype embryos. Midline is to the left. A-E: Triple immunofluorescence for Olig3, Mash1, and Shh. Mash1 is expressed in two progenitor domains; one is the prethalamic ventricular zone (B, arrow) and the other is a separate domain located more caudally (B, arrowhead). This second domain overlaps with the Olig3 expression domain (C). D is a higher-magnification image of $\mathrm{C}$, and shows that a majority of Mash1-expressing cells are also Olig3positive in this second domain (arrow). E shows that the small Mash1 expression domain (asterisk) is immediately caudal to the Shhexpressing ZLI (arrowheads), and does not overlap with it. We name this Mash1-positive domain pTH-R. F-J: Triple immunofluorescence for Olig3, Ngn2, and Shh. Ngn2 is expressed in a large progenitor domain in the thalamus ( $\mathrm{G}$, double arrows) and a small, separate domain located more rostroventrally (G, arrow). Both domains overlap with Olig3 $(\mathrm{H})$. I is a higher-magnification image of $\mathrm{H}$, and shows that a majority of Ngn2-expressing cells are also Olig3-positive (I, arrows). J shows that the small, Ngn2-expressing domain matches the

Shh-expressing ZLI (J, arrowheads). Note that the Mash1-positive domain pTH-R is negative for both Ngn2 and Shh (J, asterisk). We name the Ngn2-positive thalamic progenitor domain pTH-C (Fig. 9). F and $\mathrm{G}$ also show that the habenular ventricular zone (asterisk) is negative for Olig3 and positive for Ngn2. K-O: Triple immunofluorescence for Olig3, Nkx2.2, and Shh. Nkx2.2 is expressed in two separate bands (L, arrowhead and thin arrow) of progenitor cells, as well as in the mantle zone (L, double arrows). The caudodorsal domain overlaps with Olig3 (M). N is a higher-magnification image of M. Most of Nkx2.2-expressing cells are also Olig3-positive ( $\mathrm{N}$, arrow). O shows that Nkx2.2 is expressed at a very low level in the ZLI (arrowheads). Nkx2.2 expression is much higher in the domain immediately caudal to the ZLI, which coincides with pTH-R (O, asterisk). P-R: The relationship between Olig3 and Mash1 expression is already established at E10.5. Arrowhead in Q shows the pTH-R domain, which expresses both Olig3 and Mash1. S-X: The same is true for Olig3 and Ngn2 (S-U), as well as for Olig3 and Nkx2.2 (V-X). Scale bars $=100$ $\mu \mathrm{m}$ for D,E,I,J,N,O; $200 \mu \mathrm{m}$ for other panels.

the endogenous Olig3 protein is degraded upon differentiation of progenitor cells. This feature has been successfully used to analyze the lineage of Olig3-expressing progenitor cells in the spinal cord (Muller et al., 2005).

At E11.0 $\beta$-gal expression is the same as endogenous Olig3 (Fig. 4A-C, arrows). At E12.0 $\beta$-gal and Olig3 heavily overlap in most of the thalamic ventricular zone (Fig. 4D-F, arrow). At this stage the mantle zone expresses a much higher level of $\beta$-gal than Olig3 (Fig. 4F, double arrows), which is consistent with the greater stability of the $\beta$-gal protein. At E16.5 all the cortex-

projecting thalamic nuclei express $\beta$-gal (Fig. 4G-J). We also find that the habenula (Fig. 4G; HB) and the pretectum (Fig. 4I; PT) are not contributed by Olig3-expressing progenitors. This is compatible with the lack of Olig3 expression in the habenular and the pretectal ventricular zone (Fig. 2A,Q). Comparison of $\beta$-gal expression with nuclear DAPI staining at higher magnification images (Fig. 4K) shows that more than $95 \%$ of the cells in cortexprojecting thalamic nuclei are $\beta$-gal-positive. Therefore, it is unlikely that there is significant lineage mixing between the pretectum/habenula and the thalamus. 

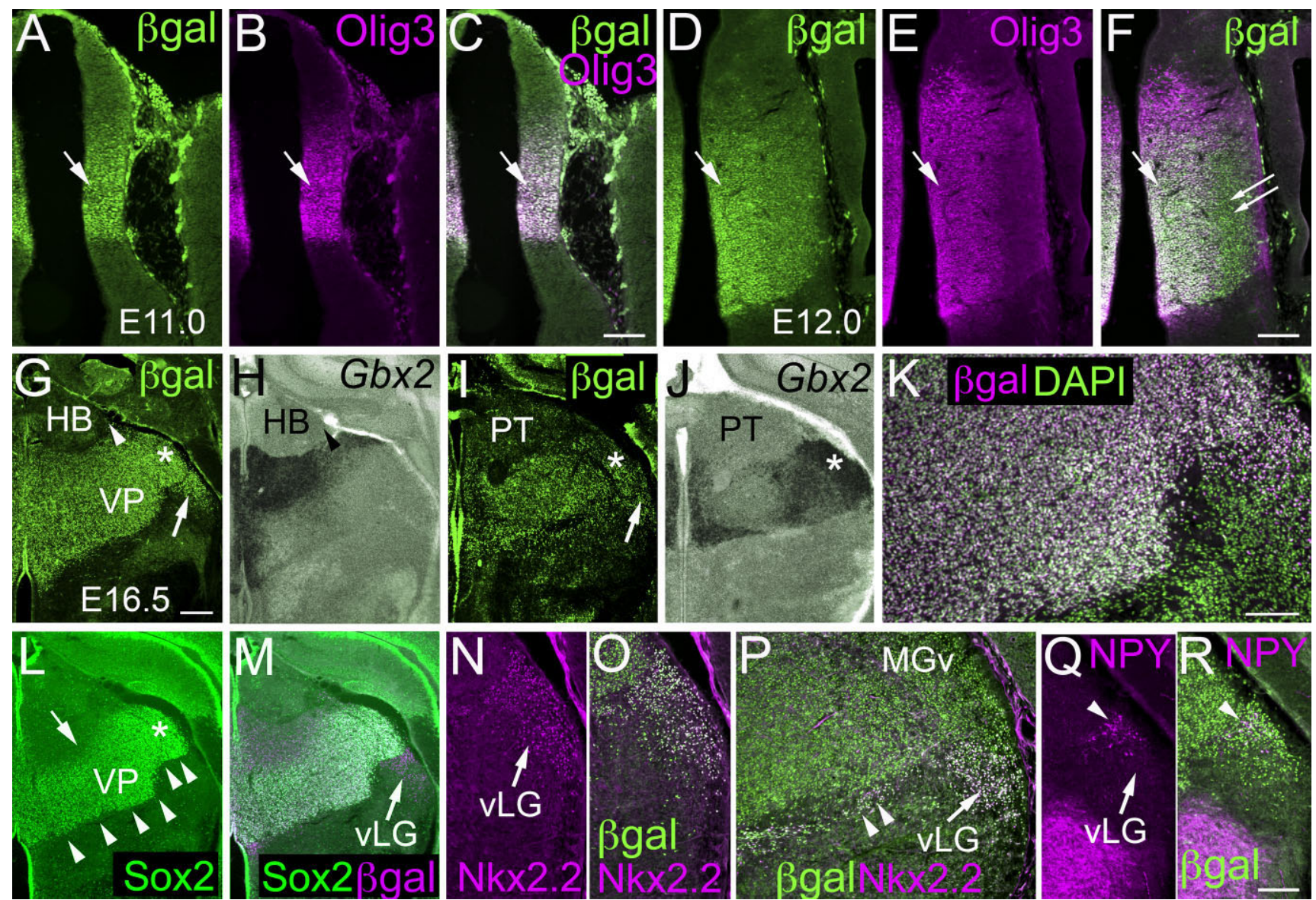

Fig. 4. Lineages of Olig3-expressing thalamic and ZLI progenitor cells. Frontal sections of E11.0 (A-C), E12.0 (D-F), and E16.5 (G-R) diencephalon of Olig3 $3_{F P-I R E S n l s l a c Z /+}$ embryos show the lineage of Olig3-expressing progenitors. A-C: At E11.0, $\beta$-gal expression driven by the Olig3 locus (A) matches the endogenous Olig3 expression (B). $\mathrm{C}$ shows an overlap between $\beta$-gal and Olig3 expression in the thalamus (arrow). D-F: At E12.0, $\beta$-gal and Olig3 expression still overlaps in the ventricular zone (arrow), but in the mantle zone where differentiated neurons predominate we detect more $\beta$-gal than Olig3, reflecting the disappearance of endogenous Olig3 protein $(\mathrm{F}$, double arrows). G-J: At E16.5 $\beta$-gal expression (G,I) is found broadly in the thalamus. Expression pattern of $G b x 2(\mathrm{H}, \mathrm{J})$ is shown for comparison. Sections in $\mathrm{G}$ and $\mathrm{H}$ are adjacent to each other and are more rostrodorsal than I and J, which are also adjacent sections. $\beta$-Gal is detected in all the nuclei in the thalamus, including VP, dLG (G, asterisk) and $\mathrm{MGv}$ (I, asterisk), but not in the habenula $(\mathrm{G}, \mathrm{Hb})$ or in the pretectum (I, PT). The region lateral to the habenula ( $\mathrm{G}, \mathrm{H}$, arrowhead) is negative for $\beta$-gal and $G b x 2$. In addition to thalamic nuclei that project to the cortex, $\beta$-gal is expressed more rostrally (G,I, arrow). K: Compar- ison of the nuclear DAPI staining and $\beta$-gal expression at a high magnification shows that more than $95 \%$ of the cells in the thalamus are derived from Olig3-positive cells. L,M: Comparison of Sox2 and $\beta$-gal expression shows that at least part of vLG is contributed by Olig3-expressing progenitor cells. Sox2 is expressed at higher levels in rostral thalamic nuclei including VP and dLG (L, asterisk) than in caudodorsal nuclei (L, arrow). Sox2 expression has a sharp rostral boundary (L, arrowheads). N-R: Further analysis of Olig3 ${ }^{+}$ progenitor-derived cells at E16.5. $\mathrm{N}$ and $\mathrm{O}$ show that Nkx2.2 overlaps with $\beta$-gal in vLG. $\mathrm{P}$ is a more caudoventral section than $\mathrm{N}$ and $\mathrm{O}$, and shows that $\beta$-gal is expressed in ${ }^{2 L G}$ and the cells between the thalamus and the prethalamus ( $\mathrm{P}$, arrowheads). The $\beta$-gal-positive cells in vLG also express $\mathrm{Nkx2.2}$. Q and $\mathrm{R}$ (same level as $\mathrm{N}$ and $\mathrm{O}$ ) show that some of the Olig3-progenitor derived cells are positive for neuropeptide Y (NPY), which marks intergeniculate leaflet (Q,R, arrowhead). Note that only some of the $\beta$-gal-expressing cells in vLG are NPY-positive. Scale bars $=100 \mu \mathrm{m}$ for K,N-R; $200 \mu \mathrm{m}$ for other panels.
Some of the $\beta$-gal-positive cells (Fig. 4G,I, arrow) appeared to be located rostral to the cluster of sensory nuclei of the thalamus including the dorsal lateral geniculate (dLG, Fig. 4G, asterisk) and the ventral part of medial geniculate ( $\mathrm{MGv}$, Fig. 4I, asterisk). This region is part of the ventral lateral geniculate (vLG), which has been traditionally designated as a prethalamic (or ventral thalamic) nucleus instead of thalamic (or dorsal thalamic) nucleus. We then compared the distribution of $\beta$-gal with that of the high-mobility-group (HMG) box transcription factor Sox2. Sox2 is expressed throughout mouse embry- ogenesis in neural progenitors of the central nervous system (Uwanogho et al., 1995; Uchikawa et al., 2003). In addition, Sox 2 is expressed in the mantle zone of embryonic chick thalamus (Uchikawa et al., 1999, 2003; Hashimoto-Torii et al., 2003). We find that Sox2 is expressed in the mantle zone of the mouse thalamus (Fig. $4 \mathrm{~L})$. Sox 2 expression in the postmitotic thalamus is strong in rostrally located nuclei, including ventral posterior (VP), dLG (Fig. 4G, asterisk) and MGv (not shown) nuclei, with a sharp rostral boundary (Fig. 4L, arrowheads). The cluster of $\beta$-gal-positive cells that are rostral to dLG and 
MGv is in the Sox2-negative domain (Fig. 4M, vLG). To further characterize this $\beta$-gal-positive population, we used Nkx2.2 as a marker. In addition to the progenitor cells in pTH-R and the prethalamus, Nkx2.2 is expressed in part of the postmitotic vLG in mouse (Kitamura et al., 1997). Double immunostaining of $\beta$-gal and $\mathrm{Nkx} 2.2$ at different levels shows that most of the Nkx2.2-expressing vLG cells are $\beta$-gal-positive (rostral-dorsal level: Fig. $4 \mathrm{~N}-\mathrm{O}$, caudal-ventral level: Fig. 4 P).

Because some literature identifies intergeniculate leaflet (IGL) between the dLG and vLG, we examined if Olig3expressing progenitors also contributes to IGL by comparing the $\beta$-gal expression with the expression of neuropeptide Y (NPY), one of the markers for IGL (Botchkina and Morin, 1995a,b). Double immunofluorescence for $\beta$-gal and NPY reveals that IGL is derived from Olig3expressing cells (Fig. 4Q,R, arrowhead). In a more caudalventral section, $\beta$-gal-positive cells are in a band of cells that runs mediolaterally, in addition to the laterally located vLG (Fig. 4P).

In summary, Olig3-epressing progenitor cells contribute to the major part of the thalamic mantle zone that contains nuclei projecting to the cortex, as well as the IGL and the lateral part of vGL, and a band of cells that runs at the boundary between the thalamus and the prethalamus. These regions can be derived from Olig3-expressing progenitors either in the pTH-C, pTH-R, ZLI, or, with a smaller likelihood, from the prethalamic progenitors immediately rostral to the ZLI.

\section{Ngn1- and Ngn2-expressing progenitor cells contribute to all the thalamic nuclei that project to the cortex}

To further dissect the fates of progenitor cells in pTH-R, pTH-C, and the ZLI, all of which express Olig3, we analyzed the cell lineages for Ngn1, Ngn2, and Ptix2. Ngn1 and Ngn2 are both expressed in pTH-C and the ZLI (Figs. 3, 5A). Pitx2 (Muccielli et al., 1996; Kitamura et al., 1997; Martin et al., 2002) is expressed in the ZLI. We used Ngn $1^{E G F P /+}$ BAC transgenic mice (Gong et al., 2003) and Ngn $2^{\text {KIEGFPI+ }}$ knockin mice (Seibt et al., 2003). To analyze the lineage of Pitx2-expressing cells, we crossed Pitx $2^{\mathrm{Cre} /+}$ mice with transgenic mice containing a conditional Cre reporter allele that expresses nuclear-localized lac $Z$ under the control of the chicken $\beta$-actin promoter $(N$-lacZ) (Zinyk et al., 1998).

In Ngn $1^{E G F P /+}$ BAC transgenic mice, expression of EGFP in the diencephalon at E12.5 matches the endogenous Ngn1 in pTH-C and the ZLI (Fig. 5A-C, asterisk). Endogenous Ngn2 shows a similar expression pattern to that of Ngn1-driven EGFP in the ventricular zone (Fig. 5D, asterisk). Endogenous Mash1 is excluded from the Ngn1-driven EGFP-positive cells (Fig. 5E). Ngn1-driven EGFP is expressed in the mantle zone lateral to pTH-C, which no longer expresses endogenous Ngn1 (Fig. 5B, \#). Cells lateral to the ZLI are also EGFP-positive (Fig. 5B, arrowhead). These ZLI-derived cells are spatially separate from EGFP-positive cells originated from pTH-C or other progenitor domains that express Ngn1, such as those in the hypothalamus (McNay et al., 2006).

At E16.5, Ngn1-driven EGFP is expressed widely in the thalamic mantle zone (Fig. $5 \mathrm{H}$ ). The entire thalamic mantle zone expressing Sox2 is included in the EGFP expression domain (Fig. 5J). Caudodorsally, the EGFP signal extends into the thalamic nuclei that express a very low level of Sox2 (Fig. 5H,I, arrow) and further into the HB (Fig. $5 \mathrm{H}$ ), with the exception of a small wedge of cells lateral to the $\mathrm{HB}$ (Fig. $5 \mathrm{H}$, arrowhead). As we discuss below, this EGFP-negative population appears to be contributed by Mash1-positve progenitors (Fig. 5T). In summary, Ngn1-expressing progenitor cells give rise to all the thalamic nuclei that project to the cortex. Based on the spatially separated lineages between the pTH-C and ZLI, as well as the lack of major contribution to the thalamic mantle zone by progenitor cells in the habenula (Fig. 4K), it is likely that pTH-C alone contributes to the entire thalamic mantle zone projecting to the cortex.

In addition to the thalamus, strong Ngn1-driven EGFP is detected in a population of cells rostral to VP and dLG nuclei at E16.5 (Fig. 5J,K, arrow). This population is in the medial part of $\mathrm{vLG}$, and has little overlap with Nkx2.2-expressing cells in the lateral vLG (Fig. 5K, double arrow). There is also no overlap between Ngn1-driven EGFP and NPY, a marker for IGL neurons (data not shown). In a more caudal-ventral section, Ngn1-driven EGFP is not detected in Nkx2.2-expressing vLG (Fig. 5L, asterisk), but is expressed in a band of cells that runs along the border between the thalamus and the prethalamus. Thus, it is likely that Ngn1-expressing cells in the ZLI contribute to a medial part of vLG as well as cells sandwiched between the thalamus and prethalamus.

Analysis of $\mathrm{Ngn} 2^{\text {KIEGFP/+ }}$ embryos at E14.5 reveals that EGFP is expressed in most of the thalamic mantle zone, as well as in similar cell populations in vLG and the boundary between the thalamus and the prethalamus that express Ngn1-driven EGFP (Fig. 5M-R). This result complements our finding on the Ngn1 lineage. Seibt et al. (2003) analyzed the same $\mathrm{Ngn} 2^{\mathrm{KIEGFP/+}}$ mice at postnatal day 1 (P1) and showed that EGFP is expressed only in "rostral" thalamic nuclei, instead of all the nuclei that project to the cortex. The difference between our result at E14.5 and the result by Seibt et al. at P1 may be due to the difference in the developmental stages analyzed. At E14.5 EGFP signal should come from both Ngn2-expressing progenitor cells in the ventricular zone and Ngn2-expressing postmitotic cells in the mantle zone, whereas at P1 EGFP driven by Ngn2 expression in progenitor cells may have degraded already, and the "rostral" nuclei with the EGFP signal are those that still express Ngn2 mRNA at a high level in postmitotic cells (Nakagawa and O'Leary, 2001; Gonzalez et al., 2002; Seibt et al., 2003). In contrast, there is less complexity in the Ngn1 lineage, because, unlike Ngn2 mRNA, Ngn1 mRNA is expressed at a very low level in the thalamic mantle zone (data not shown).

With regard to the fates of the ZLI cells, because Ngn1 and Ngn2 are both expressed in the progenitor cells in pTH-C and the ZLI, and cells in the mantle zone of each domain become close to each other at E14.5 or later (e.g., compare the two regions shown by arrow and double arrow in Fig. 5R), it is still possible that progenitor cells in pTH-C give rise to the medial vLG and cells between the thalamus and the prethalamus. Thus, we analyzed the lineage of Pitx2-positive cells. Ptix2 (also known as Brx1 or Otlx2) is a homeobox transcription factor expressed in the ZLI, but apparently not in the thalamic, prethalamic, or prethalamic ventricular zone (Muccielli et al., 1996; Kitamura et al., 1997; Martin et al., 2002). Pitx2 is not expressed in dividing cells in the diencephalon, but appears to have a small overlap with Shh in the ZLI (Kita- 


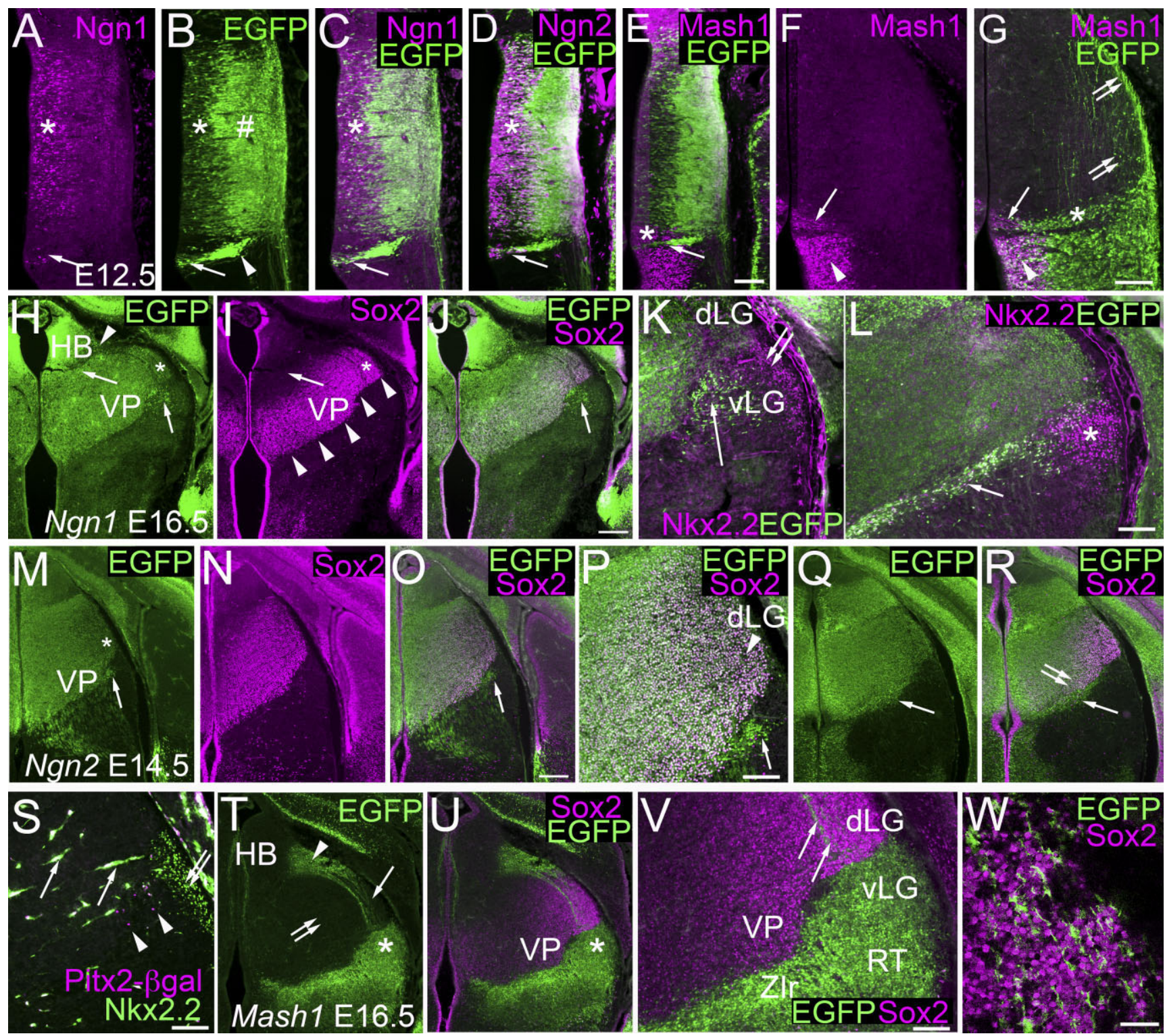

Fig. 5. Lineages of Mash1-, Ngn1-, Ngn2-, and Pitx2-expressing cells in the thalamus and the ZLI. Frontal sections of $N g n 1^{E G F P /+}$ embryos at E12.5 (A-E), and E16.5 (H-L), Ngn2 $2^{\text {KIEGFP }}$ embryos at E14.5 (M-R), and Mash1 $1^{E G F P /+}$ embryos at E12.5 (F,G) and E16.5 (T-W) show the lineages of Ngn1-, Ngn2-, and Mash1-expresssing progenitor cells. Lineage of Pitx2-expressing cells in the ZLI at E14.5 is also shown (S). Midline is to the left. A-E: Endogenous Ngn1 (A) and EGFP derived from the $N g n 1^{E G F P}$ allele (B) match in the thalamic ventricular zone (A-C, asterisk) and in the $\mathrm{ZLI}(\mathrm{A}-\mathrm{C}$, arrow). The thalamic mantle zone $(\mathrm{B}, \#)$ expresses EGFP but not endogenous Ngn1. D shows that Ngn2 and Ngn1 are expressed in similar patterns in the progenitor domain pTH-C (D, asterisk) and in the ZLI (D arrow). Mash1 expression in pTH-R (E, asterisk) has no overlap with Ngn1-driven EGFP in the thalamus or in the ZLI. F,G: Endogenous Mash1 (F) and EGFP derived from the Mash $1^{E G F P}$ allele (G) match in the pTH-R domain (F,G, arrow) and in the prethalamic ventricular zone (F,G, arrowhead). Mash1-driven EGFP is also expressed in the mantle zone immediately lateral to the Mash1-positive progenitor domains (G, asterisk for pTH-R). In addition, Mash1-driven EGFP is found in cell bodies and processes within the thalamus (G, double arrows). H-L: At E16.5 progeny of Ngn1-expressing progenitor cells are distributed in most of the thalamus, including VP and dLG $(\mathrm{H}$ asterisk), as well as in the habenula (H, HB). Ngn1-driven EGFP is not detected in a small wedge-shaped region lateral to the habenula $(\mathrm{H}$, arrowhead). Double staining with Sox2 shows that there is a small population of EGFP-positive cells (H,J, arrow) beyond the rostral boundary of Sox2 expression ( $\mathrm{I}$, arrowheads). $\mathrm{K}$ is a highmagnification image of the dLG/vLG region, showing that Ngn1driven EGFP is expressed in the medial part of $\mathrm{vLG}$ ( $\mathrm{K}$, arrow). This population has minimal overlap with $\mathrm{Nkx} 2.2$ in vLG (K, double arrows). At a more caudoventral level (L), EGFP is not detected in vLG, but is expressed in cells between the thalamus and prethalamus $(\mathrm{L}$, arrow). Nkx2.2 is expressed in both vLG (L, asterisk) and the cells between the thalamus and the prethalamus (L, arrow). M-R: At E14.5 progeny of Ngn2-expressing cells are found in all the cortexprojecting thalamic nuclei, including VP and dLG (M, asterisk). In addition, EGFP is expressed in a small population in the prethalamus (M,O,P, arrow), in a similar manner to Ngn1-driven EGFP (H, arrow). At a higher magnification, EGFP and Sox2 are colocalized in all the thalamic nuclei projecting to the cortex, including dLG $(\mathrm{P})$. In a more caudoventral level, Ngn2-driven EGFP is expressed in the cells between the thalamus and the prethalamus, outside the Sox2expressing domain (Q,R, arrow). At this level the thalamic mantle zone ( $R$, double arrow) and the EGFP-positive band of cells between the thalamus and the prethalamus (R, arrow) are juxtaposed to each other. S: At E14.5, Pitx2-driven $\beta$-gal is expressed in the medial part of vLG (arrowhead). Nkx2.2 expression is found in the lateral vLG (double arrow). Artifact staining is marked by arrows. T-W: At E16.5 progeny of Mash1-expressing progenitor cells are distributed broadly in the vLG (T,U, asterisk), but not significantly within the cortexprojecting part of the thalamus. The region lateral to the habenula is strongly labeled with EGFP (T, arrowhead). V is a high-magnification image of dLG/vLG region showing the mutual exclusion between Sox2 and EGFP and a fiber-like EGFP labeling in the lateral part of the thalamus, especially within $\mathrm{dLG}$ (V, arrows). W is a confocal image of dLG showing that the EGFP label within dLG is not colocalized with Sox2-expressing cell bodies. Scale bars $=200 \mu \mathrm{m}$ for A-J,M-O,Q-R; $100 \mu \mathrm{m}$ for $\mathrm{K}, \mathrm{L}, \mathrm{P}, \mathrm{S}, \mathrm{V} ; 50 \mu \mathrm{m}$ for $\mathrm{W}$. 
mura et al., 1997; Martin et al., 2002). Therefore, we expect that the lineage of Pitx2-expressing cells is at least part of the cell lineage derived from progenitor cells in the ZLI. Kitamura et al. reported that at E14.5 and E18 Pitx2 is expressed in the medial-caudal part of vLG and external medullary lamina (eml), the term for the thalamusprethalamus boundary often used in adult brains. Mucchielli et al. suggest that the Pitx2 expression is detected in dorsal zona incerta (ZId) and reticular thalamic nucleus at E16.5. These gene expression data do not provide definitive information about the lineage of cells expressing Pitx2, but still suggest that the ZLI may later contribute to these regions in the mantle zone.

In embryos heterozygous for both Pitx $2^{\text {Cre }}$ and the $N$-lac $Z$ reporter alleles, we detect $\beta$-gal-positive cells in the medial part of vLG (Fig. 5S, arrowheads) and a band of cells between the thalamus and prethalamus (not shown), which are similar to the region that arises from Ngn1- and Ngn2-positive progenitor cells. Since Pitx2 is not expressed in pTH-C, pTH-R or the prethalamus (Muccielli et al., 1996; Kitamura et al., 1997; Martin et al., 2002 ), it is unlikely that progenitors in the thalamus or the prethalamus contribute to medial vLG or cells at the boundary between the thalamus and the prethalamus. Conversely, it is likely that the progeny of the ZLI cells is restricted to these two regions, without migrating to the thalamus or the prethalamus. These two regions are also positive for Olig3-driven $\beta$-gal (Fig. 4N-P). Because Olig3 is expressed in the ZLI (Fig. 2M-O), results of Olig3-, Ngn1-, Ngn2-, and Pitx2-lineage tracing data are consistent with each other.

\section{pTH-R progenitor cells contribute to ventral lateral geniculate nucleus, but not to thalamic nuclei projecting to the cortex}

We next analyzed the lineage of Mash1-expressing progenitors by examining the distribution of EGFP in Mash $1^{E G F P /+}$ mice. Mash1 is expressed in pTH-R and the prethalamus, but not in pTH-C or the ZLI. At E12.5 Mash $1^{E G F P /+}$ brains show broad EGFP expression in the prethalamic ventricular zone and pTH-R, both of which match the distribution of endogenous Mash1 (Fig. 5F,G, arrowhead and arrow). EGFP in the mantle zone extends laterally from pTH-R (Fig. 5F, asterisk) and the prethalamic ventricular zone, leaving a blank region in between that may correspond to the lineage of the ZLI. Interestingly, we see EGFP signal within the thalamic mantle zone at E12.5, especially along the lateral surface (Fig. 5G, double arrows). The EGFP signal includes cell bodies, together with processes, suggesting the presence of cells tangentially migrating dorsal- and caudal-wards either from pTH-R-derived cells or the prethalamus.

In the E16.5 diencephalon EGFP expression is strong in vLG, ZI, and reticular nucleus (RT) (Fig. 5T,V). In the thalamic mantle zone medial to the dLG nucleus EGFP signal is very sparse (Fig. 5T, double arrow). Within dLG (Fig. 5T, arrow) we detect EGFP signal reminiscent of that found at E12.5 (compare Fig. 5G, double arrows, and T, arrow), but few cell bodies are EGFP-positive. Confocal analysis (Fig. 5W) shows that in dLG there is no colocalization of EGFP signal with nuclear staining for Sox2, which is expressed in postmitotic cells most likely derived from the pTH-C domain (Fig. 5I,N,U). We also detect no
Mash1-driven EGFP signal within thalamocortical axons (not shown).

Thus, unlike the progenitor cells in the pTH-C domain, Mash1-positive progenitor cells in pTH-R do not appear to make a significant contribution to the thalamic neurons projecting to the cortex. By comparing the expression patterns of endogenous Olig3 and Mash1, as well as the lineages of Olig3- and Mash1-expressing progenitor cells, it is likely that the lateral part of vLG and IGL are derived from pTH-R, which expresses both Olig3 and Mash1. However, a low level of Olig3 expression in the prethalamus (Fig. 3) leaves a possibility that some of the lateral vLG and IGL cells are derived from the prethalamic progenitor cells as well.

Further caudally and dorsally, strong EGFP signal in both soma and processes is found in a cell mass lateral to the habenula, near the stria medullaris (Fig. 5T, arrowhead). This region is strongly GABA-positive at E16.5 (not shown) and is negative for Gbx2 expression as well as Olig3-driven and Ngn1-driven EGFP (Figs. 4G, 5H, arrowhead). Thus, this parahabenular mass of cells rich in GABA are likely to have originated from either pTH-R or the prethalamic progenitors, and have tangentially migrated within the thalamic mantle zone.

If all the thalamic nuclei projecting to the cortex arise from pTH-C, not from pTH-R, then how is the diversity of these thalamic nuclei established? In the next section, we asked if pTH-C is a homogeneous population that gives rise to all the cortex-projecting thalamic nuclei, or if there are multiple subdomains within pTH-C, each of which produces different sets of nuclei. For this purpose, we analyzed the expression patterns of two additional transcription factors, Dbx1 and Olig2.

\section{Homeobox gene Dbx1 is expressed in the anterior pretectum and in a caudal-to- rostral gradient within the thalamic progenitor domain pTH-C}

Dbx1 is a homeodomain transcription factor expressed in restricted regions of the developing central nervous system. Shoji et al. (1996) reported that in the early embryonic mouse diencephalon, $D b x 1$, is expressed in the thalamus and rostral half of the pretectum, with an increasing level away from the ZLI. This led us to perform detailed analysis of both mRNA and protein expression in order to determine if Dbx1 is expressed differentially within the thalamic progenitor domain pTH-C (Fig. 6). First, we analyzed $D b x 1$ expression by in situ hybridization together with markers that delineate the thalamus (Olig3) and caudal pretectum (Lhx1) (Fig. 6A-C). Lhx1 is a LIM-homeodomain transcription factor and is expressed in the mantle zone of the caudal pretectum (Suda et al., 2001). Dbx1 overlaps with Olig3 only in the caudal-dorsal part of the thalamus (Fig. $6 \mathrm{~A}, \mathrm{~B}$, brackets), and that $D b x 1$ and $L h x 1$ do not seem to overlap in the pretectum (Fig. $6 \mathrm{~A}, \mathrm{C})$, suggesting that $D b x 1$ is expressed in the rostral pretectum and the caudal-dorsal thalamus. We also examined the expression of the bHLH gene BHLHB4 and Mab21L1. BHLHB4 is expressed in the pretectum, immediately caudal to $G b \times 2$-expressing thalamic mantle zone (Bramblett et al., 2002). Mab21L1 is one of the two mouse homologs of Mab21, a gene expressed in the developing chick pretectum (Vieira et al., 2005). We find that BHLHB4 and Mab21L1 are both expressed in the mantle 
zone of the rostral pretectum, overlapping with $D b x 1$, although $D b x 1$ is expressed in the ventricular zone whereas BHLHB4 and Mab21L1 are in the mantle zone (Fig. 6D,E). These results confirm that $D b x 1$ is expressed in the ventricular zone of both the rostral pretectum and the thalamus.

We next did double immunofluorescence analysis with anti-Dbx1 antibody in order to determine the expression pattern more thoroughly. At E11.5 in a rostral-dorsal section (type a section shown in Fig. 1), Dbx1 is found at a high level in the ventricular zone of the HB (Fig. 6F). Expression extends rostrally and ventrally into the thalamus, with a lower level toward the ZLI. Double immunofluorescence with Olig3 allowed us to plot the fluorescence intensity of the Dbx1 labeling within and around the thalamus (Fig. 6I). We find that there is a caudodorsalhigh to rostroventral-low gradient of Dbx1 expression within the thalamic ventricular zone. Near the ZLI expression is at the background level (Fig. 6F,I), but there is another expression domain in the prethalamus immediately rostral to the ZLI (Fig. 6F, arrowhead). Analysis of type caudoventral sections at E11.5 together with Olig3 and Pax7 shows that Dbx1 expression is in the thalamus and in the rostral pretectum, but not in the caudal pretectum (Fig. 6J-N). The same result is obtained at E10.5 (Fig. 6O-V).

In sagittal sections at E11.5, we also find that Dbx1 is highly expressed in the pretectum and caudal thalamus (Fig. 9). Within the thalamus the level of expression is

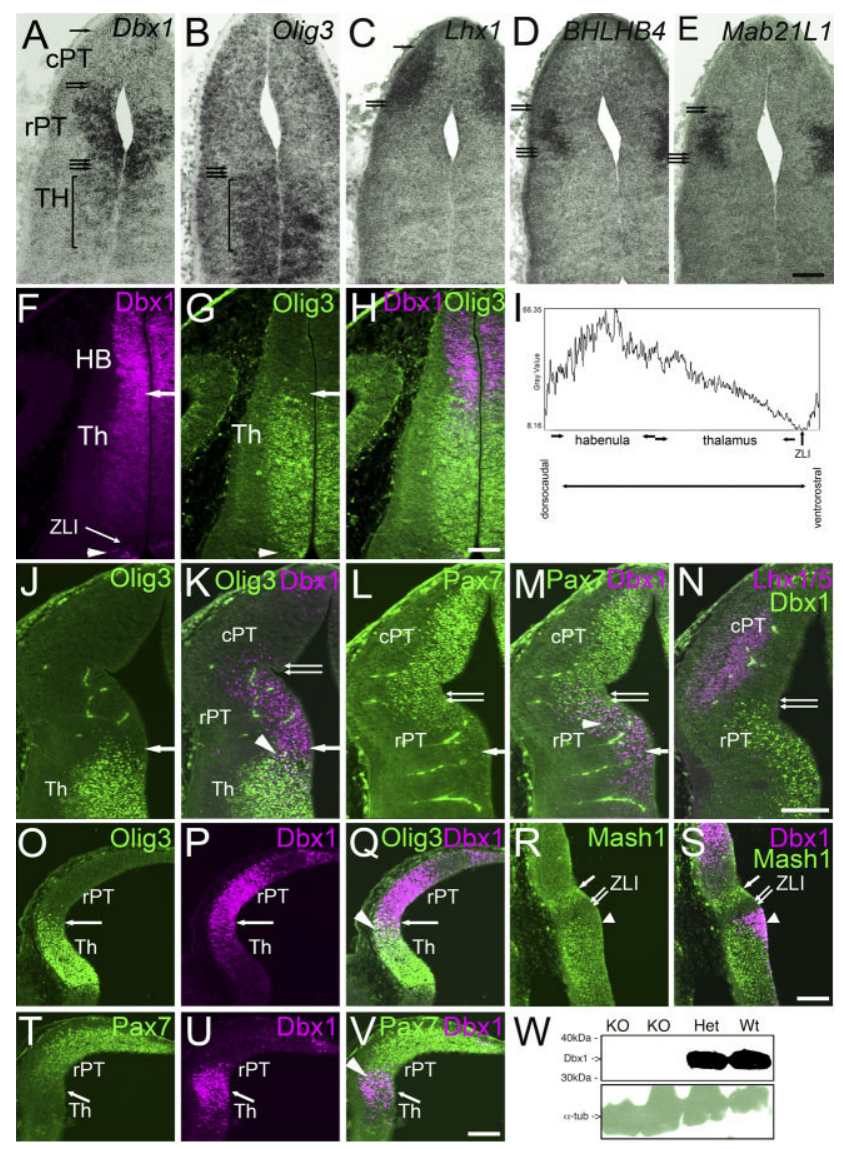

higher in the more dorsal-caudal region compared to the more ventral-rostral region (Fig. $9 \mathrm{~F}-\mathrm{H}$ ).

In summary, Dbx1 is expressed in a high-caudodorsal to low-rostroventral gradient within the thalamic progenitor domain pTH-C. Dbx1 is also expressed in the rostral pretectum and habenula, as well as in the most caudal part of the prethalamus. This expression pattern suggests that Dbx1-expressing progenitor cells may contribute preferentially to postmitotic thalamic cells that are located away from the ZLI and the basal plate, and that Dbx1 might function in specifying the positional identity of the thalamic nuclei.

\section{Dbx1-expressing progenitor cells contribute mainly to thalamic nuclei away from the sources of Shh}

From the graded expression pattern of Dbx1 within pTH-C, we hypothesized that Dbx1-expressing progenitor cells preferentially give rise to caudally and dorsally located thalamic nuclei that are away from the sources of Shh, the ZLI, and the basal plate. Thus, we analyzed mice heterozygous for the $D b x 1^{\text {nlsLac }}$ allele (Pierani et al., 2001). Short-term lineage tracing with $D b x 1^{\text {nlsLacZ/+ }}$ has been done to analyze the fate of Dbx1-expressing progen-

Fig. 6. Dbx1 is expressed in a caudal-to-rostral gradient in the thalamic ventricular zone. Frontal sections of the diencephalon of wildtype E11.5 (A-H,J-N) and E10.5 (O-V) mouse embryos. Midline is to the left. A-E: In situ hybridization on E11.5 adjacent sections for Dbx1 (A), Olig3 (B), Lhx1 (C), BHLHB4 (D), and Mab21L1 (E). Dbx1 is strongly expressed in the rostral part of the pretectum (A, rPT), and weakly in the caudal part of the thalamus (A, TH). The caudal pretectum does not express $D b x 1$ (A, cPT). Markers that delineate these regions are Olig3 (B; thalamic ventricular zone), Lhx1 (C; caudal pretectal mantle zone), BHLHB4 and Mab21L1 (D,E; rostral pretectal mantle zone). F-H: Double immunofluorescence for Dbx1 and Olig3 shows that Dbx1 protein is expressed at a high level of in the habenula (F, HB), and the expression extends into the thalamus in a high-caudodorsal to low-rostroventral gradient (F). In addition, Dbx1 is expressed immediately rostroventral to the ZLI (F, arrowhead). I: Quantification of fluorescence intensity shows that Dbx1 protein is expressed in a smooth, caudodorsal-to-rostroventral gradient within the thalamic ventricular zone. J-N: Double immunofluorescence with other markers confirms that at E11.5, Dbx1 expression is detected in the caudal thalamic ventricular zone $(\mathrm{J}, \mathrm{K})$, as well as in the rostral pretectum $(\mathrm{L}, \mathrm{M})$ but not in the caudal pretectum $(\mathrm{N})$. J and $\mathrm{K}$ show overlap between Dbx1 and Olig3 in the thalamus ( $\mathrm{K}$, arrowhead). Boundaries of the rostral pretectum are indicated by arrow and double arrow $(\mathrm{J}-\mathrm{N})$. L and M show that Pax7 is expressed in the entire pretectal ventricular zone, and Dbx1 partially overlaps with Pax7 in the rostral part (M, arrowhead). N shows that Lhx1/5 is detected in the caudal pretectal mantle zone, similar to the $L h x 1$ mRNA shown in C. Lhx1/5 and Dbx1 do not overlap at all. Single and double arrows indicate boundaries of the rostral pretectum. O-V: A similar expression pattern for Dbx1 is already found at E10.5. Dbx1 expression overlaps with Olig3 in the thalamus (Q, arrowhead), and with Pax7 in the pretectum (V, arrowhead). Note that double immunofluorescence with Mash1 indicates that the rostral expression domain of Dbx1 ( $\mathrm{S}$, arrowhead) is immediately rostral to the ZLI (R,S, double arrows). The Mash1-expressing pTH-R domain is shown in single arrows (R,S). In $\mathrm{O}-\mathrm{Q}$ and $\mathrm{T}-\mathrm{V}$, boundary between the rostral pretectum and the thalamus is indicated by arrow. W: Western blot analysis to show the specificity of anti-Dbx1 antibody. The antibody detects a single band of a $35-\mathrm{kD}$ protein in heterozygous $D b x 1^{\text {LacZ }}$ (Het) and wildtype (Wt) brains, but not in $D b x 1^{\text {LacZ }}$ null (KO) brains. Each lane represents the data from midbrain and caudal diencephalon of a single E12.5 embryo of the same litter. Loading controls with anti- $\alpha$-tubulin antibody is shown below each lane. Scale bars $=200 \mathrm{~mm}$. 

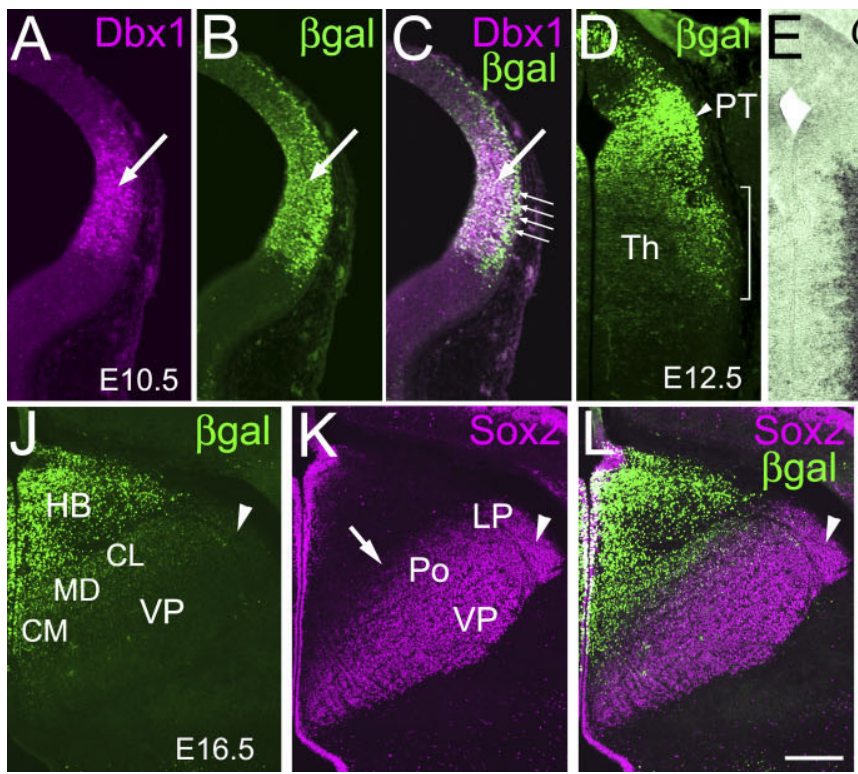

Fig. 7. Dbx1-expressing progenitor cells contribute only to the caudal thalamic nuclei. Frontal sections of the diencephalon of $D b x 1^{\text {LacZI+ }}$ embryos showing the lineage of Dbx1-expressing progenitors. Midline is to the left. A-C: Double immunofluorescence for endogenous Dbx1 (A), $\beta$-gal (B), and merge (C) at E10.5. Large arrows in $\mathrm{A}-\mathrm{C}$ show the colocalization of Dbx1 and $\beta$-gal in the pretectal/ thalamic region. Small arrows in $\mathrm{C}$ show cells the in emerging mantle zone that are derived from Dbx1-positive progenitors but have lost expression of Dbx1 protein. D-I: Lineage of Dbx1-expressing progenitor cells at E12.5. D is immunofluorescence for $\beta$-gal and $\mathrm{E}$ is in situ hybridization for $G b x 2$, on adjacent sections. Brackets in D and $\mathrm{E}$ show that some Dbx1-positive progenitors contribute to Gbx2 positive, postmitotic cells in the thalamus. F-I are double immunofluorescence for $\beta$-gal and markers for the pretectum and the thalamus. Pax7 is expressed in the entire pretectal ventricular zone, and $\beta$-gal overlaps with $\operatorname{Pax} 7$ (F, asterisk). Lhx1/5 is expressed only in the caudal pretectal mantle zone, where $\beta$-gal is not expressed $(G$, aster-

itor cells in the spinal cord (Pierani et al., 2001; Lanuza et al., 2004). In $D b x 1^{\text {nlsLacZl+ }}$ mice we detect no changes in the expression of regional markers compared to wildtype mice (data not shown).

At E10.5, Dbx1 and $\beta$-gal are colocalized in the progenitor cells of pretectal-thalamic region (Fig. 7A,B, arrow). Some of the earliest-born neurons in the mantle zone have already lost the expression of Dbx1 but still maintain $\beta$-gal expression (Fig. 7C, small arrows). We find similar results at E11.5 and E12.5 (data not shown). We next asked if postmitotic cells derived from Dbx1-expressing progenitor cells are restricted to certain thalamic nuclei. We analyzed the distribution of $\beta$-gal and other markers of the thalamus on the same or adjacent sections. At E12.5, expression of Gbx2 is detected in a large part of the thalamic mantle zone (Fig. 7E). Part of the thalamic mantle zone expresses both Gbx2 and $\beta$-gal (Fig. 7D,E, bracket). Thus, Dbx1-expressing progenitor cells do contribute to cells in the thalamic mantle zone. Double staining of $\beta$-gal and Pax7 detects an overlap in the pretectal ventricular zone (Fig. 7F, asterisk), confirming that the region located rostrally and ventrally to this overlapping population is in the thalamic ventricular zone (Fig. $7 \mathrm{~F}$, arrow). On the other hand, $\beta$-gal and Lhx1/5 barely overlap, demonstrating that Dbx1-expressing progenitors contribute to rostral

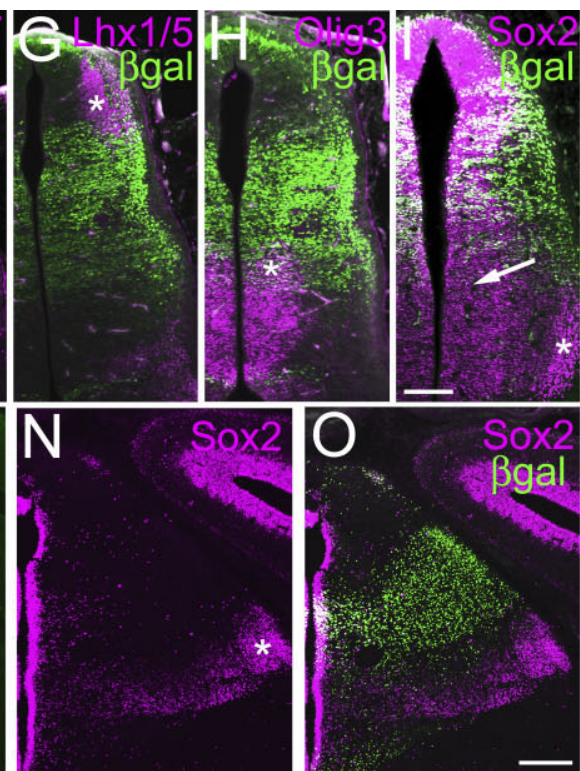

isk). $\mathrm{H}$ shows that $\beta$-gal overlaps with Olig3 in the caudal thalamic ventricular zone (asterisk). Sox2 is expressed ubiquitously in the ventricular zone (I, arrow), and preferentially in the rostral part of the thalamic mantle zone ( $\mathrm{I}$, asterisk). $\beta$-gal is not colocalized with Sox 2 in the mantle zone. J-O: Double immunofluorescence showing the lineage of Dbx1-positive progenitors at E16.5. J-L are more caudodorsal than M-O. $\beta$-gal-positive nuclei include central medial (CM), mediodorsal (MD), and caudal lateral (CL) (J). These nuclei are located caudodorsally in the thalamus, and are negative for Sox 2 K, arrow). Sox2 is expressed strongly in the rostral thalamic nuclei including dLG (K, arrowhead), ventral posterior (VP), lateral posterior (LP), posterior ( $\mathrm{Po}$ ), and $\mathrm{MGv}(\mathrm{N}$, asterisk), and few $\beta$-gal-positive cells are found in these nuclei (J-M). The habenula (J, HB) as well as the rostral part of the pretectum (M, arrow) express high levels of $\beta$-gal, but the more caudal part of the pretectum (M, double arrow) does not. Scale bars $=200 \mu \mathrm{m}$.

pretectal mantle zone, not the caudal one that expresses Lhx1/5 (Fig. 7G, asterisk). As expected, $\beta$-gal and Olig3 are colocalized in the caudal thalamic progenitor cells (Fig. 7H). At late embryonic stages, Sox2 is expressed preferentially in the rostral thalamic mantle zone (see Figs. 4L, 5I,N,U). At E12.5 Sox2 is expressed in progenitor cells across the entire thalamus (Fig. 7I, arrow) as well as postmitotic cells in the rostral-ventral part of the thalamus (Fig. 7I, asterisk). Interestingly, this postmitotic Sox2 does not colocalize with $D b x 1$-driven $\beta$-gal (Fig. 7I), suggesting that only caudal-dorsal cells in the thalamic mantle zone are derived from Dbx1-expressing progenitors.

At E16.5 $\beta$-gal is expressed in the habenula (Fig. 7J) and the rostral part of the pretectal mantle zone (Fig. 7M). Robust expression of $\beta$-gal is found in the caudal-dorsal part of the thalamus, including central lateral (CL), mediodorsal (MD), and central medial (CM) nuclei (Fig. 7J). This part includes very few cells that express Sox2 (Fig. $7 \mathrm{~K})$. In contrast, more rostrally located thalamic nuclei that express high levels of Sox2, such as posterior ( $\mathrm{Po}$, lateral posterior (LP), dLG, and VP, have only a very small number of $\beta$-gal-expressing cells (Fig. 7K,L, arrowhead shows dLG). In a more caudal-ventral section (Fig. 7M-O), $\beta$-gal is found in rostrally located nuclei of the pretectum (Fig. 7M, arrow), but not in a rostrally and ventrally 


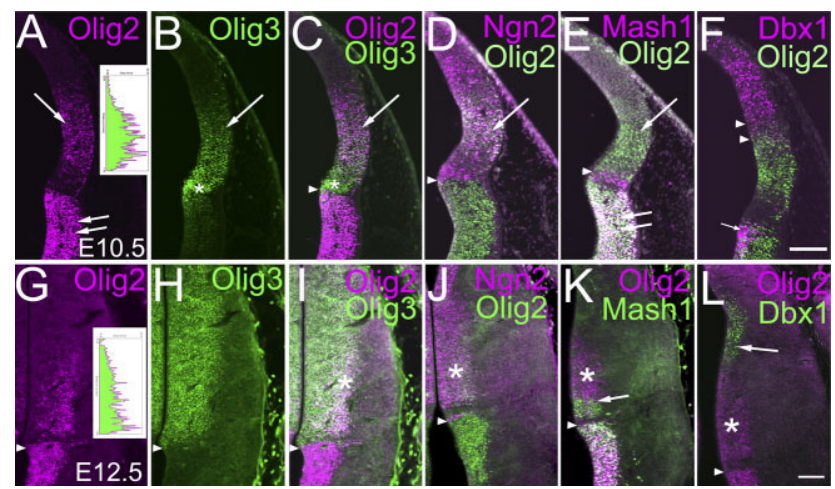

Fig. 8. Olig2 is expressed preferentially in the rostral part of pTH-C domain. Frontal sections of wildtype E10.5 (A-F) and E12.5 (G-L) mouse embryos. Midline is to the left. A-F: At E10.5, Olig2 is expressed in the prethalamic (A, double arrows) and the thalamic (A, arrow) ventricular zones. Expression in the thalamus is higher rostroventrally than caudodorsally (A, inset). This is similar to Olig3 (B), but Olig3 is also expressed in pTH-R and ZLI, where Olig2 is not expressed (B, asterisk). Merge of A and B clearly shows the difference between Olig2 and Olig3 (C). Arrowhead in C and D is a boundary between the ZLI and the prethalamus. Double immunofluorescence for Olig2 and Ngn2 shows that both of these two proteins are expressed in pTH-C (D, arrow). Mash1 and Olig2 overlap in the prethalamus (E, double arrows) but not in the thalamus, where Mash1 is expressed only in pTH-R (E, arrowhead) and Olig2 is expressed only in pTH-C (E, arrow). Dbx1 and Olig2 do not overlap within pTH-C (F). Dbx1 is expressed at a higher level in the caudodorsal part, whereas Olig2 is higher in the rostroventral part, forming a boundary region, where expression levels of both proteins taper off ( $\mathrm{F}$, between arrowheads). G-L: Olig2 expression pattern at E12.5 is similar to that of E10.5. Olig2 shows high-rostroventral to low-caudodorsal gradient in pTH-C ( $\mathrm{G}$, inset). Arrowheads in G-L show the boundary between the ZLI and the prethalamus. Olig2 and Olig3 expression significantly overlaps in pTH-C, especially near the lateral part of the ventricular zone (I, asterisk). Ngn2 and Olig2 overlap in pTH-C (J, asterisk). In K, Mash1 (arrow) and Olig2 (asterisk) do not overlap in the thalamus. In L, Dbx1 (arrow) and Olig2 (asterisk) do not overlap within pTH-C. Scale bars $=200 \mu \mathrm{m}$.

located thalamic nucleus, MGv, which expresses a high level of Sox2 (Fig. 7M,N, asterisk). We conclude that pTH-C progenitor domain is heterogeneous in terms of the expression of Dbx1, and Dbx1-expressing, caudal/dorsal progenitors within pTH-C preferentially give rise to caudally and dorsally located thalamic nuclei.

\section{bHLH gene Olig2 is expressed in a rostral- to-caudal gradient in the thalamic progenitor zone}

Finally, we examined the expression of the bHLH protein Olig2 (Lu et al., 2000; Takebayashi et al., 2000; Zhou et al., 2000). In the diencephalic ventricular zone, Olig2 is expressed at a high level in the prethalamus and at a lower level in the thalamus (Zhou et al., 2000; Takebayashi et al., 2002). We find that Olig2 expression within the thalamus is higher in the rostral-ventral part than in the caudal-dorsal part at E10.5 (Fig. 8A, inset), E11.5 (not shown) and E12.5 (Fig. 8G, inset). This graded pattern is similar to that of Olig3 at E10.5. However, by E12.5 Olig3 expression becomes ubiquitous within the entire thalamic ventricular zone, whereas Olig2 expression is still much higher in the rostral-ventral part of the thalamus, and this patterns stays until E14.5 (not shown). Double immu-
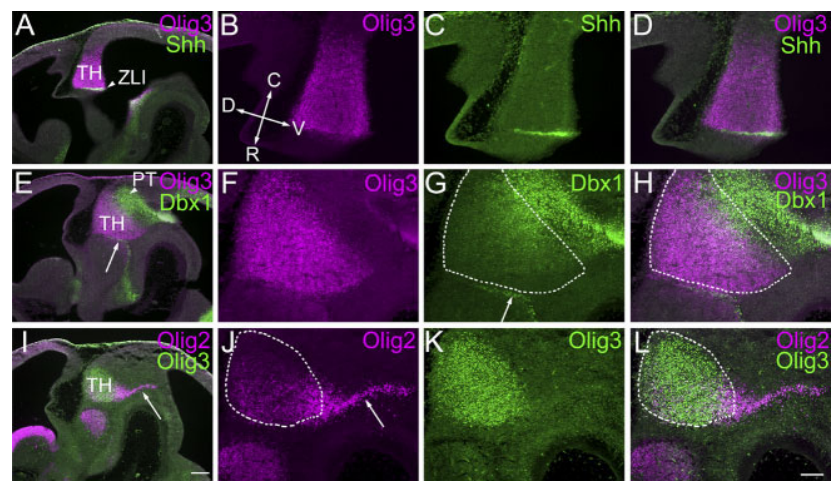

Fig. 9. Sagittal sections showing the expression patterns of Olig3, Shh, Dbx1, and Olig2 at E11.5. Sagittal sections of wildtype E11.5 mouse embryos. A-D, E-H, and I-L are from the same sections, respectively. Telencephalon is on the left and midbrain is on the right. The rostrocaudal and dorsoventral axes of the diencephalon are curved, but at the level of the ZLI, dorsal is almost to the left and rostral is almost to the bottom (arrows shown in B). A-D: Olig3 is highly expressed in the thalamus ( $\mathrm{TH}$ in A). The ZLI, which is positive for Shh, is located at the rostral end of the Olig3-expression domain (ZLI in A). Overlapping expression of Olig3 and Shh is shown at a higher magnification (B-D). E-H: Dbx1 is expressed in the caudaldorsal part of the thalamus. Olig3 defines the thalamus and ZLI (F), and the outline of the thalamus is shown in dotted lines in $\mathrm{G}$ and $\mathrm{H}$. Dbx1 expression is detected in the caudal and dorsal part within the dotted line. $\mathrm{H}$ shows the partial overlap between Olig3 and Dbx1. Arrows in $\mathrm{E}$ and $\mathrm{G}$ shows the Dbx1 expression immediately rostral to the ZLI, which is also shown in Figure 6. I-L: Olig2 is expressed in the rostral-ventral part of the thalamus. Olig3 defines the thalamus and ZLI (K), and the outline of the thalamus is shown in dotted lines in J and L. L shows the partial overlap between Olig3 and Olig2. Olig2 expression continues on to the ventral part of the caudal diencephalon (arrows in I and J). Scale bars $=200 \mu \mathrm{m}$ for A,E,I; $100 \mu \mathrm{m}$ for other panels.

nofluorescence of Olig2 and Mash1 shows that Olig2 is not expressed in Mash1-positive pTH-R (Fig. 8E,K). Thus, the rostral limit of Olig2 expression in the thalamus matches the rostral limit of pTH-C (Fig. 8D,E,J,K). In addition, within pTH-C, Olig2, and Dbx1 show reciprocal expression patterns at both E10.5 and E12.5 (Fig. 8F,L).

In sagittal sections at E11.5, Olig2 expression level is high in the rostral-ventral part of the thalamus, whereas the level is very low in the more caudal and dorsal part (Fig. 9I-L).

In summary, we detect two distinct gene expression domains in the thalamic ventricular zone caudal to the ZLI; the large, caudal domain, pTH-C, expresses Ngn1 and Ngn2 and contributes to the entire thalamic mantle zone projecting to the cortex. The smaller, rostral domain, pTH-R, expresses Mash1 and Nkx2.2, and this domain is likely to contribute to a set of retinorecipient thalamic nuclei, IGL and lateral vLG, neither of which is known to project to the cortex. Within pTH-C, Dbx1 and Olig2 are expressed preferentially in the caudal-dorsal and rostralventral parts, respectively, and Dbx1-expressing progenitors generate mostly caudally and dorsally located thalamic nuclei. Together, these data clearly demonstrate the positional heterogeneity of progenitor cells within the thalamus.

\section{DISCUSSION}

Despite the importance of the thalamus and the thalamocortical projections in the development of animal 
behavior, little is known about the heterogeneity of thalamic progenitor cells or how such heterogeneity contributes to the generation of a diverse set of distinct nuclei at later developmental stages. In this study we combined the analysis of protein/gene expression with short-term lineage tracing using transgenic mice to characterize the progenitor domains and their fates in the mouse thalamus.

Although a definitive conclusion awaits the lineage tracing studies using genes that are restricted to only one of the three progenitor domains, pTH-C, pTH-R, or ZLI, our comparison of lineages of Olig3-, Mash1-, Ngn1-, Ngn2-, and Pitx2-positive cells suggests that pTH-C and pTH-R contribute to distinct domains of the postmitotic thalamus. pTH-C, which express Olig3, Ngn1, and Ngn2, contributes to nuclei that project to the cortex. These are the nuclei that have been traditionally designated as thalamic nuclei in mammals (Jones, 2007). The Olig3- and Mash1positive pTH-R, on the other hand, likely contributes to IGL and the lateral vLG, both of which have retinal afferents but have no efferent projections to the cortex. It is also possible that pTH-R gives rise to the GABAergic cells located lateral to the habenula. The progenitors within the ZLI itself are likely to contribute to the medial part of vLG, as well as a band of cells interspaced between the thalamus and the prethalamus.

\section{Possible mechanisms that set up the differential transcription factor expression in the thalamic ventricular zone}

How are the differential expression patterns of transcription factors established in the dividing progenitor cells of the thalamus? Molecular mechanisms for this regulation are currently unknown, except that Nkx2.2 has been shown to be induced by Shh (Kiecker and Lumsden, 2004; Vieira et al., 2005; Vieira and Martinez, 2006). Remarkably, however, the early embryonic thalamus is surrounded by a number of signaling molecules, suggesting that they may play crucial roles in regulating the expression of these transcription factors (Echevarria et al., 2003; Lim and Golden, 2007). For example, Shh is expressed ventrally and rostrally to the thalamus in the basal plate and the ZLI, respectively. In addition to Shh, the ZLI also expresses Fgf8 and Wnt8b. Dorsal to the thalamus a variety of signaling molecules including BMPs are expressed in the roof plate. Numerous studies have indicated that each of these molecules play a role in thalamic patterning, but the precise roles of these factors in controlling gene expression in dividing progenitor cells in the ventricular zone is not well understood.

Although Shh, Wnts, BMPs, and FGFs control the gene expression in progenitor cells of numerous brain and body regions throughout the developing embryo, including the dorsal and ventral spinal cord, retina, cerebral cortex, as well as the limb, the unique positioning of these molecules within and around the thalamus makes a direct comparison with other regions difficult. Nonetheless, the ventral spinal cord and the thalamus share a remarkable set of transcription factors being expressed during early embryogenesis. For example, Nkx2.2 and Mash1 are expressed in immediate proximity to the source of Shh, and Ngn2 and Olig2 are expressed in the next domain. Dbx1 is found further away from Shh. In the ventral spinal cord, expression of some of these proteins is controlled by differential activity of Shh signaling. Specifically, the Gli family of transcription factors are shown to be involved in transducing the Shh gradient into graded control of transcription in the progenitor cells. Similar mechanisms involving Gli proteins have been proposed for the patterning of the chick thalamus in the regulation of Sox14 and Gbx2 (Hashimoto-Torii et al., 2003). Although both Sox14 and Gbx2 are expressed postmitotically in the mantle zone, not in progenitor cells in the ventricular zone, it is conceivable that graded Shh signaling first establishes the heterogeneity of dividing progenitor cells by controlling the expression of the transcription factors that we analyzed in this study, and then such heterogeneity is inherited to postmitotic cells, leading to differential expression of Sox14 and Gbx2. However, as we discuss below, Shh signaling could also operate directly on postmitotic cells.

With the similarities discussed above, however, there are also many striking differences between the thalamus and the ventral spinal cord in terms of the patterns of gene expression. One such difference is in the pattern of Olig3 expression. Olig3 is expressed in the entire progenitor domains of the thalamus as well as in the ZLI, whereas in the spinal cord its expression is restricted to specific interneuron progenitor domains. Another noticeable difference is that the expression of Dbx1 and Olig2 does not form as distinct boundaries in the thalamus as in the ventral spinal cord, and that this pattern persists throughout neurogenesis in the thalamus. These differences are likely to be caused by contribution of other signaling molecules than Shh, or by differential transduction mechanisms of Shh expression gradient into the regulation of gene expression. For example, in the spinal cord the Gro/TLE family of transcriptional corepressors are expressed when distinct gene expression boundaries are being established. Muhr et al. (2001) showed that these corepressors play a key role in establishing abrupt gene expression boundaries (Muhr et al., 2001). Thus, it is possible that within pTH-C, Gro/TLE-mediated suppression does not occur in the same manner as in the ventral spinal cord, and this may lead to the appearance of less distinct gene expression boundaries within pTH-C.

\section{Revised definition of the mammalian thalamic mantle zone}

Although we do not have lineage tracing data for a gene that is expressed exclusively in pTH-R, combined analysis of different transgenic lines indicates that the thalamic progenitor domain pTH-R is likely to give rise to IGL and lateral vLG. The locations of these nuclei immediately next to the cortex-projecting thalamic nuclei also make it reasonable to consider that progenitors in pTH-R migrate radially and contribute to IGL and lateral vLG. These nuclei have traditionally been classified as prethalamic (or ventral thalamic), instead of thalamic (or dorsal thalamic) nuclei in the literature of mammalian neuroanatomy and embryology (Jones, 2007), presumably because of their locations and the lack of neurons in these nuclei that project to the cortex. IGL is distinguished from vLG by its involvement in the control of circadian rhythm and its nontopographic nature of the retinal afferents (Botchkina and Morin, 1995a,b; Harrington, 1997). Thus, our results appear to contradict the widely accepted definition of the mammalian (dorsal) thalamus in that a thalamic progenitor domain, pTH-R, contributes to IGL and the lateral part of vLG. 
Interestingly, detailed immunohistochemical studies on the developing chick thalamus support our finding. They have proposed that the most rostrally located neuroepithelial cells expressing cadherin $6 \mathrm{~B}$ give rise to the anteroventral subregion of the thalamus, which later develops into internal nucleus of the optic tract (ITO) and perirotundic area (ApR) (Puelles et al., 1991; Uchikawa et al., 1999; Redies et al., 2000; Yoon et al., 2000). Fate mapping analysis using quail-chick grafts is also consistent with this proposal (Garcia-Lopez et al., 2004). ITO and $A p R$ are located between the avian equivalents of dLG and $\mathrm{vLG}$, express Nkx2.2, and receives nontopographic retinal input and send projections to the midbrain. All these features are shared by the mammalian IGL (Martinez et al., 1991; Martinez-de-la-Torre et al., 2002). Taken together, these independent studies clearly indicate that the thalamic ventricular zone not only gives rise to cortex-projecting nuclei, but also to nuclei that were previously thought to belong to noncortex projecting, prethalamic nuclei. Thus, we propose that from the perspective of the embryonic origin, IGL and lateral vGL should be defined as thalamic nuclei.

In addition to IGL and lateral vLG, we found that pTH-R may contribute to another cell population that is located at the dorsal-caudal end of the thalamus lateral to the habenula. This region is negative for $G b x 2$ (Fig. $4 \mathrm{H}$, arrowhead) and is immunoreactive for GABA (not shown). The fact that this population expresses Mash1-driven EGFP (Fig. 5T, arrowhead) but not Ngn1-driven EGFP (Fig. 5H, arrowhead) suggests that it is derived from pTH-R. Moreover, there are EGFP-positive cell bodies and processes in the lateral part of the thalamus at E12.5 (Fig. $5 \mathrm{G})$, raising the possibility that these cells are generated rostrally in pTH-R and tangentially migrate within the mantle zone in a caudal-dorsal direction to occupy the location near the habenular border. In fact, studies of cell migration in the chick diencephalon using retrovirus vectors have observed a number of cells migrating tangentially outside of the ventricular zone, which is consistent with this possibility (Golden and Cepko, 1996; Golden et al., 1997), as are the results of chick-quail graft experiments (Garcia-Lopez et al., 2004). Based on the location and proposed manner of tangential migration from rostralmost thalamic neuroepithelium in the chick, this Mash1-driven population might be equivalent to the parvocellular superficial nucleus (SPC) (Radies et al., 2000), or the superficial microcellular nucleus (SMC), which is negative for $G b x 2$ and located superficial to SPC (Radies et al., 2000; Martinez-de-la-Torre et al., 2002).

However, since Mash1 is expressed in the prethalamic ventricular zone in addition to pTH-R, we cannot exclude the possibility that this parahabenular cell mass (SPC/ SMC) is derived from the prethalamus. The lack of a major contribution by Olig3-positive progenitors to this domain (Fig. 4G, arrowhead) favors this second possibility unless we hypothesize heterogeneity of progenitor cells within the pTH-R domain, which does seem to exist based on our gene expression analysis (Fig. 3D); for example, it is possible that Mash1-positive, Olig3-negative cells in pTH-R specifically contribute to the parahabenular cell mass, and that Olig3-positive cells in pTH-R give rise to IGL and lateral vLG.

\section{Cell lineage of the ZLI}

Our analysis of $N g n 1^{E G F P}, N g n 2^{\text {KIEGFP }}$, and Pitx $2^{\text {Cre }}$ mice indicates that the Shh-expressing ZLI gives rise to cells in the medial part of vLG as well as the band of cells between the thalamus and the prethalamus. Previous reports showed that Pitx2/Brx1/Otlx2 is expressed immediately lateral to the Shh-expressing ZLI at E11.5 in the mouse (Muccielli et al., 1996; Kitamura et al., 1997; Martin et al., 2002). At later embryonic stages, this gene is expressed in the mantle zone. Kitamura et al. (1997) described the expression domain as medial part of vLG and external medullary lamina, whereas Mucchielli et al. (1996) show that the Pitx2 expression is detected in dorsal zona incerta (ZId) and reticular nucleus at E16.5. The progeny of the ZLI cells based on our lineage analysis is similar to what Kitamura et al. described as cells expressing Pitx2 at late embryonic stage, although we acknowledge that a distinction between the ZId and the cell band between the thalamus and the prethalamus (which may correspond to cells in the eml) is difficult, with a lack of definitive markers for ZI subdivisions at embryonic stages (Kolmac and Mitrofanis, 1999; Mitrofanis, 2005). Keyser (1972) described a cellular mass "located at the place of the former tract of the zona limitans," which lies between the thalamus and the prethalamus, and named it the interstitial nucleus of the tract of the zona limitans. The ZLI-derivative that we propose in this study is likely to correspond to this nucleus, although it is unclear if Keyser's terminology exactly refers to the population we identified.

Baek et al. (2006) recently showed that the ZLI has strong expression of the repressor-type bHLH factor Hes1 at E10.5, and that Hes1 represses Mash1 expression in the ZLI. They also showed that persistent expression of Hes1 in neural progenitor cells reduce cell proliferation and neurogenesis, which implies that it is unlikely that many Hes1-expressing cells in the ZLI proliferate or differentiate. Therefore, although it had already been implicated from gene expression analyses (Muccielli et al., 1996; Kitamura et al., 1997; Martin et al., 2002), it was surprising that our lineage analysis showed that ZLI could give rise to postmitotic cells in the diencephalon. We speculate that Hes1 expression is downregulated in the ZLI after E10.5, which would allow the ZLI progenitor cells to express Ngn1 and Ngn2 and differentiate into cells in vLG and ZId.

\section{Molecular heterogeneity within pTH-C domain and mechanisms of thalamic nuclei specification}

In this study we found that only one distinct progenitor domain, pTH-C, contributes to all the thalamic nuclei that send robust projections to the cortex. The opposing graded expression of Dbx1 and Olig2 within pTH-C clearly indicates that there is an extremely heterogeneous pool of progenitor cells that could contribute to the diversity of thalamic nuclei. The fact that Dbx1-expressing progenitor cells preferentially give rise to cells in caudal and dorsal thalamic nuclei provides strong evidence for this idea. Differential thresholds for enhancers of target genes of graded transcription factors such as Dbx1 or Olig2 are likely to give rise to cell populations with distinct gene expression profiles (Stathopoulos and Levine, 2005). Although we have not completely ruled out the possibility 
that there are distinct subdomains within pTH-C, or the possibility that there are temporal identities within progenitor cells that might control the serial generation of different cell types in the thalamus (Pearson and Doe, 2004), the results in our current study make it likely that the process of cell type specification in the thalamus continues beyond the stage when progenitor cells become postmitotic. Such postmitotic mechanisms could include direct actions of signaling molecules such as Shh on postmitotic cells, and cell-cell interactions between postmitotic cells, such as those mediated by Notch-Delta signaling (Peng et al., 2007) or cadherins (Suzuki et al., 1997; Redies et al., 2000; Yoon et al., 2000).

In this respect, proposals for dividing the thalamic mantle zone into secondary subdivisions, each of which would be later parcellated into distinct nuclei, is noteworthy (Davila et al., 2000; Radies et al., 2000; Gonzalez et al., 2002). Nevertheless, we do not find clear correlation between their subdivisions and our gene expression and lineage analyses, except that their anteroventral subregion in the chick seems to correspond to the lineage of pTH-R that we described in this study.

It is likely that specification of thalamic cell types and nuclei is a complex process in which multiple mechanisms cooperatively control different aspects at different developmental stages until the mature nuclear organization emerges. Although we still do not know the detailed molecular basis for this process, our current study clearly demonstrates that specification starts at the progenitor cell stage and that the progenitor heterogeneity sets the stage for subsequent steps of specification.

\section{Technical considerations of lineage tracing}

There are some intrinsic limitations with the lineage tracing method used in this study. First, when a recombinase-based system is not used to permanently mark progenitor cells, marker genes such as EGFP or $\beta$-gal that are "knocked-into" the gene locus of interest will eventually be degraded and become undetectable, potentially leading to underestimation of cell fates. To reduce this possibility, we did lineage tracing analysis on multiple developmental stages, aiming not to miss the cell populations that express the marker protein only transiently. With this method we found that in most cases marker expression started to decline only at postnatal stages, suggesting that analysis of progenitor cell fates in the thalamus needs to be done at embryonic stages.

The second limitation is that when the gene to be analyzed is expressed in more than one progenitor domains that are close to each other or even next to each other, it is difficult to designate the fates of progenitor cells in each domain. To circumvent this problem, we again analyzed the marker protein at multiple developmental stages to see if there is a sign of cell mixing between the progeny of more than one separate domain. In addition, we used a combination of transgenic mice, each of which marks the descendents of different combinations of progenitor domains.

As discussed above, there are still difficulties in interpreting lineage data when massive tangential migration within the mantle zone is expected. Nevertheless, we think the genetic lineage tracing methods described here will provide invaluable tools in developmental biology. The increased availability of gene knockin mice and BAC EGFP transgenic mice developed by GENSAT and other organizations will further enhance the efficiency of largescale fate mapping.

\section{CONCLUSION}

The present study has revealed molecular heterogeneity in the thalamic progenitors cells and demonstrated that this heterogeneity contributes to the generation of different nuclei in the postmitotic thalamus. This opens a number of new ways to genetically visualize the thalamocortical projections from specific thalamic nuclei and to manipulate different aspects of thalamic development. In order to fully understand the mechanisms for the specification of thalamic nuclei, further studies will be necessary to clarify the roles of the transcription factors that we have analyzed in this study.

\section{ACKNOWLEDGMENTS}

We thank Thomas Müller for Olig3 ${ }^{\text {GFP-IRESnlslacZ }}$ mice, Alessandra Pierani, Guillermo Lanuza, Tomoko Velásquez, and Martyn Goulding for Dbxi $1^{\text {nlslac } Z}$ mice, François Guillemot for Ngn2 $2^{\text {KIEGFP }}$ embryos, and Gene Expression Nervous System Atlas (GENSAT) for $N g n 1^{E G F P}$ and Mash $1^{E G F P}$ mice. Ngn1 $1^{E G F P}$ mice were generated by Jane Johnson's laboratory. We thank Developmental Studies Hybridoma Bank (DSHB)/NICHD/ University of Iowa, David Anderson, Gavin Chapman, Jane Johnson, Thomas Müller, Masato Nakafuku, Naoki Takahashi, and Hirohide Takebayashi for antibodies and cDNAs, Paul Marker for confocal analysis, and Naoko Koyano-Nakagawa for help in Western blot analysis, discussion, and critical reading of the article.

\section{LITERATURE CITED}

Angevine JBJ. 1970. Time of neuron origin in the diencephalon of the mouse. An autoradiographic study. J Comp Neurol 139:129-187.

Baek JH, Hatakeyama J, Sakamoto S, Ohtsuka T, Kageyama R. 2006. Persistent and high levels of Hes1 expression regulate boundary formation in the developing central nervous system. Development 133: 2467-2476.

Botchkina GI, Morin LP. 1995a. Organization of permanent and transient neuropeptide Y-immunoreactive neuron groups and fiber systems in the developing hamster diencephalon. J Comp Neurol 357:573-602.

Botchkina GI, Morin LP. 1995b. Specialized neuronal and glial contributions to development of the hamster lateral geniculate complex and circadian visual system. J Neurosci 15:190-201.

Bramblett DE, Copeland NG, Jenkins NA, Tsai MJ. 2002. BHLHB4 is a bHLH transcriptional regulator in pancreas and brain that marks the dimesencephalic boundary. Genomics 79:402-412.

Braun MM, Etheridge A, Bernard A, Robertson CP, Roelink H. 2003. Wnt signaling is required at distinct stages of development for the induction of the posterior forebrain. Development 130:5579-5587.

Briscoe J, Novitch BG. 2007. Regulatory pathways linking progenitor patterning, cell fates and neurogenesis in the ventral neural tube. Philos Trans R Soc Lond B Biol Sci (in press).

Bulfone A, Puelles L, Porteus MH, Frohman MA, Martin GR, Rubenstein JL. 1993. Spatially restricted expression of Dlx-1, Dlx-2 (Tes-1), Gbx-2, and Wnt-3 in the embryonic day 12.5 mouse forebrain defines potential transverse and longitudinal segmental boundaries. J Neurosci 13: 3155-3172.

Davila JC, Guirado S, Puelles L. 2000. Expression of calcium-binding proteins in the diencephalon of the lizard Psammodromus algirus. J Comp Neurol 427:67-92.

Echelard Y, Epstein DJ, St-Jacques B, Shen L, Mohler J, McMahon JA, McMahon AP. 1993. Sonic hedgehog, a member of a family of putative signaling molecules, is implicated in the regulation of CNS polarity. Cell 75:1417-1430. 
Echevarria D, Vieira C, Gimeno L, Martinez S. 2003. Neuroepithelia secondary organizers and cell fate specification in the developing brain. Brain Res Brain Res Rev 43:179-191.

Fode C, Ma Q, Casarosa S, Ang SL, Anderson DJ, Guillemot F. 2000. A role for neural determination genes in specifying the dorsoventral identity of telencephalic neurons. Genes Dev 14:67-80.

Garcia-Lopez R, Vieira C, Echevarria D, Martinez S. 2004. Fate map of the diencephalon and the zona limitans at the 10-somites stage in chick embryos. Dev Biol 268:514-530.

Ge W, He F, Kim KJ, Blanchi B, Coskun V, Nguyen L, Wu X, Zhao J, Heng JI, Martinowich K, Tao J, Wu H, Castro D, Sobeih MM, Corfas G, Gleeson JG, Greenberg ME, Guillemot F, Sun YE. 2006. Coupling of cell migration with neurogenesis by proneural bHLH factors. Proc Natl Acad Sci U S A 103:1319-1324.

Golden JA, Cepko CL. 1996. Clones in the chick diencephalon contain multiple cell types and siblings are widely dispersed. Development 122:65-78.

Golden JA, Zitz JC, McFadden K, Cepko CL. 1997. Cell migration in the developing chick diencephalon. Development 124:3525-3533.

Gong S, Zheng C, Doughty ML, Losos K, Didkovsky N, Schambra UB Nowak NJ, Joyner A, Leblanc G, Hatten ME, Heintz N. 2003. A gene expression atlas of the central nervous system based on bacterial artificial chromosomes. Nature 425:917-925.

Gonzalez G, Puelles L, Medina L. 2002. Organization of the mouse dorsal thalamus based on topology, calretinin immunostaining, and gene expression. Brain Res Bull 57:439-442.

Gradwohl G, Fode C, Guillemot F. 1996. Restricted expression of a novel murine atonal-related bHLH protein in undifferentiated neural precursors. Dev Biol 180:227-241.

Harrington ME. 1997. The ventral lateral geniculate nucleus and the intergeniculate leaflet: interrelated structures in the visual and circadian systems. Neurosci Biobehav Rev 21:705-727.

Hashimoto-Torii K, Motoyama J, Hui CC, Kuroiwa A, Nakafuku M, Shimamura K. 2003. Differential activities of Sonic hedgehog mediated by Gli transcription factors define distinct neuronal subtypes in the dorsal thalamus. Mech Dev 120:1097-1111.

Helms AW, Johnson JE. 2003. Specification of dorsal spinal cord interneurons. Curr Opin Neurobiol 13:42-49.

Jansen AS, Nguyen XV, Karpitskiy V, Mettenleiter TC, Loewy AD. 1995. Central command neurons of the sympathetic nervous system: basis of the fight-or-flight response. Science 270:644-646.

Jessell TM. 2000. Neuronal specification in the spinal cord: inductive signals and transcriptional codes. Nat Rev Genet 1:20-29.

Jones EG. 2001. The thalamic matrix and thalamocortical synchrony. Trends Neurosci 24:595-601.

Jones EG. 2007. The thalamus. Cambridge, UK: Cambridge University Press.

Jones EG Rubenstein JL. 2004. Expression of regulatory genes during differentiation of thalamic nuclei in mouse and monkey. J Comp Neurol 477:55-80.

Kaufman MH. 1992. Atlas of mouse development. New York: Academic Press

Keyser A. 1972. The development of the diencephalon of the Chinese hamster. An investigation of the validity of the criteria of subdivision of the brain. Acta Anat Suppl (Basel) 59:1-178.

Kiecker C, Lumsden A. 2004. Hedgehog signaling from the ZLI regulates diencephalic regional identity. Nat Neurosci 7:1242-1249.

Kitamura K, Miura H, Yanazawa M, Miyashita T, Kato K. 1997. Expression patterns of Brx1 (Rieg gene), Sonic hedgehog, Nkx2.2, Dlx1 and Arx during zona limitans intrathalamica and embryonic ventral latera geniculate nuclear formation. Mech Dev 67:83-96.

Kolmac C, Mitrofanis J. 1999. Distribution of various neurochemicals within the zona incerta: an immunocytochemical and histochemical study. Anat Embryol (Berl) 199:265-280.

Lanuza GM, Gosgnach S, Pierani A, Jessell TM, Goulding M. 2004. Genetic identification of spinal interneurons that coordinate left-right locomotor activity necessary for walking movements. Neuron 42:375-386.

Lim Y, Golden JA. 2002. Expression pattern of cLhx2b, cZic1 and cZic3 in the developing chick diencephalon. Mech Dev 115:147-150.

Lim Y, Golden JA. 2007. Patterning the developing diencephalon. Brain Res Rev 53:17-26.

Lim Y, Cho G, Minarcik J, Golden J. 2005. Altered BMP signaling disrupts chick diencephalic development. Mech Dev 122:603-620.

Liu W, Selever J, Lu MF, Martin JF. 2003. Genetic dissection of Pitx2 in craniofacial development uncovers new functions in branchial arch morphogenesis, late aspects of tooth morphogenesis and cell migration. Development 130:6375-6385.

Lo L, Dormand E, Greenwood A, Anderson DJ. 2002. Comparison of the generic neuronal differentiation and neuron subtype specification functions of mammalian achaete-scute and atonal homologs in cultured neural progenitor cells. Development 129:1553-1567.

Lu QR, Yuk D, Alberta JA, Zhu Z, Pawlitzky I, Chan J, McMahon AP, Stiles CD, Rowitch DH. 2000. Sonic hedgehog-regulated oligodendrocyte lineage genes encoding bHLH proteins in the mammalian central nervous system. Neuron 25:317-329.

Martin DM, Skidmore JM, Fox SE, Gage PJ, Camper SA. 2002. Pitx2 distinguishes subtypes of terminally differentiated neurons in the developing mouse neuroepithelium. Dev Biol 252:84-99.

Martinez S, Alvarado-Mallart RM, Martinez-de-la-Torre M, Puelles L. 1991. Retinal and tectal connections of embryonic nucleus superficialis magnocellularis and its mature derivatives in the chick. Anat Embryol (Berl) 183:235-243.

Martinez-de-la-Torre M, Garda AL, Puelles E, Puelles L. 2002. Gbx2 expression in the late embryonic chick dorsal thalamus. Brain Res Bull 57:435-438

Matsunaga E, Araki I, Nakamura H. 2001. Role of Pax3/7 in the tectum regionalization. Development 128:4069-4077.

McNay DE, Pelling M, Claxton S, Guillemot F, Ang SL. 2006. Mash1 is required for generic and subtype differentiation of hypothalamic neuroendocrine cells. Mol Endocrinol 20:1623-1632.

Mitrofanis J. 2005. Some certainty for the "zone of uncertainty"? Exploring the function of the zona incerta. Neuroscience 130:1-15.

Miyashita-Lin EM, Hevner R, Wassarman KM, Martinez S, Rubenstein JL. 1999. Early neocortical regionalization in the absence of thalamic innervation. Science 285:906-909.

Muccielli ML, Martinez S, Pattyn A, Goridis C, Brunet JF. 1996. Otlx2, an Otx-related homeobox gene expressed in the pituitary gland and in a restricted pattern in the forebrain. Mol Cell Neurosci 8:258-271.

Muhr J, Andersson E, Persson M, Jessell TM, Ericson J. 2001. Grouchomediated transcriptional repression establishes progenitor cell pattern and neuronal fate in the ventral neural tube. Cell 104:861-873.

Muller T, Anlag K, Wildner H, Britsch S, Treier M, Birchmeier C. 2005 The bHLH factor Olig3 coordinates the specification of dorsal neurons in the spinal cord. Genes Dev 19:733-743.

Nakagawa Y, O'Leary DD. 2001. Combinatorial expression patterns of LIM-homeodomain and other regulatory genes parcellate developing thalamus. J Neurosci 21:2711-2725.

Nakagawa Y, O'Leary D. 2003. Dynamic patterned expression of orphan nuclear receptor genes RORalpha and RORbeta in developing mouse forebrain. Dev Neurosci 25:233-244.

Paxinos G. 1994. Atlas of the developing rat nervous system. New York: Academic Press.

Pearson BJ, Doe CQ. 2004. Specification of temporal identity in the developing nervous system. Annu Rev Cell Dev Biol 20:619-647.

Peng CY, Yajima H, Burns CE, Zon LI, Sisodia SS, Pfaff SL, Sharma K. 2007. Notch and MAML signaling drives Scl-dependent interneuron diversity in the spinal cord. Neuron 53:813-827.

Pierani A, Moran-Rivard L, Sunshine MJ, Littman DR, Goulding M, Jessell TM. 2001. Control of interneuron fate in the developing spinal cord by the progenitor homeodomain protein Dbx1. Neuron 29:367-384.

Price M, Lazzaro D, Pohl T, Mattei MG, Ruther U, Olivo JC, Duboule D, D Lauro R. 1992. Regional expression of the homeobox gene Nkx-2.2 in the developing mammalian forebrain. Neuron 8:241-255.

Puelles L. 1995. A segmental morphological paradigm for understanding vertebrate forebrains. Brain Behav Evol 46:319-337.

Puelles L. 2001a. Thoughts on the development, structure and evolution of the mammalian and avian telencephalic pallium. Philos Trans R Soc Lond B Biol Sci 356:1583-1598.

Puelles L. 2001b. Brain segmentation and forebrain development in am niotes. Brain Res Bull 55:695-710.

Puelles L, Rubenstein JL. 1993. Expression patterns of homeobox and other putative regulatory genes in the embryonic mouse forebrain suggest a neuromeric organization. Trends Neurosci 16:472-479.

Puelles L, Rubenstein JL. 2003. Forebrain gene expression domains and the evolving prosomeric model. Trends Neurosci 26:469-476.

Puelles L, Guillen M, Martinez-de-la-Torre M. 1991. Observations on the fate of nucleus superficialis magnocellularis of Rendahl in the avian 
diencephalon, bearing on the organization and nomenclature of neighboring retinorecipient nuclei. Anat Embryol (Berl) 183:221-233.

Puelles L, Martinez S, Martinez-de-la-torre M, Rubenstein JLR. 2004. Gene maps and related histogenetic domains in the forebrain and midbrain. In: Paxinos G, ed. The rat nervous system. Amsterdam: Elsevier. p 3-25.

Redies C, Ast M, Nakagawa S, Takeichi M, Martinez-de-la-Torre M, Puelles L. 2000. Morphologic fate of diencephalic prosomeres and their subdivisions revealed by mapping cadherin expression. J Comp Neurol 421:481-514.

Seale P, Ishibashi J, Scime A, Rudnicki MA. 2004. Pax7 is necessary and sufficient for the myogenic specification of CD45+:Sca1+ stem cells from injured muscle. PLoS Biol 2:E130.

Seibt J, Schuurmans C, Gradwhol G, Dehay C, Vanderhaeghen P, Guillemot F, Polleux F. 2003. Neurogenin2 specifies the connectivity of thalamic neurons by controlling axon responsiveness to intermediate target cues. Neuron 39:439-452.

Sherman SM, Guillery. RW. 2005. Exploring the thalamus. Cambridge, MA: MIT Press.

Shimamura K, Hartigan DJ, Martinez S, Puelles L, Rubenstein JL. 1995. Longitudinal organization of the anterior neural plate and neural tube. Development 121:3923-3933.

Shoji H, Ito T, Wakamatsu Y, Hayasaka N, Ohsaki K, Oyanagi M, Kominami R, Kondoh H, Takahashi N. 1996. Regionalized expression of the Dbx family homeobox genes in the embryonic CNS of the mouse. Mech Dev 56:25-39.

Stathopoulos A, Levine M. 2005. Genomic regulatory networks and animal development. Dev Cell 9:449-462.

Suda Y, Hossain ZM, Kobayashi C, Hatano O, Yoshida M, Matsuo I, Aizawa S. 2001. Emx2 directs the development of diencephalon in cooperation with Otx2. Development 128:2433-2450.

Sun YE. 2006. Coupling of cell migration with neurogenesis by proneural bHLH factors. Proc Natl Acad Sci U S A 103:1319-1324.

Suzuki SC, Inoue T, Kimura Y, Tanaka T, Takeichi M. 1997. Neuronal circuits are subdivided by differential expression of type-II classic cadherins in postnatal mouse brains. Mol Cell Neurosci 9:433-447.

Takebayashi H, Yoshida S, Sugimori M, Kosako H, Kominami R, Nakafuku M, Nabeshima Y. 2000. Dynamic expression of basic helix-loophelix Olig family members: implication of Olig2 in neuron and oligodendrocyte differentiation and identification of a new member, Olig3. Mech Dev 99:143-148.
Takebayashi H, Ohtsuki T, Uchida T, Kawamoto S, Okubo K, Ikenaka K, Takeichi M, Chisaka O, Nabeshima Y. 2002. Non-overlapping expression of Olig3 and Olig2 in the embryonic neural tube. Mech Dev 113:169-174.

Tuttle R, Nakagawa Y, Johnson JE, O'Leary DD. 1999. Defects in thalamocortical axon pathfinding correlate with altered cell domains in Mash1-deficient mice. Development 126:1903-1916.

Uchikawa M, Kamachi Y, Kondoh H. 1999. Two distinct subgroups of Group B Sox genes for transcriptional activators and repressors: their expression during embryonic organogenesis of the chicken. Mech Dev 84:103-120

Uchikawa M, Ishida Y, Takemoto T, Kamachi Y, Kondoh H. 2003. Functional analysis of chicken Sox2 enhancers highlights an array of diverse regulatory elements that are conserved in mammals. Dev Cell 4:509-519.

Uwanogho D, Rex M, Cartwright EJ, Pearl G, Healy C, Scotting PJ, Sharpe PT. 1995. Embryonic expression of the chicken Sox2, Sox3 and Sox11 genes suggests an interactive role in neuronal development. Mech Dev 49:23-36.

Vieira C, Martinez S. 2006. Sonic hedgehog from the basal plate and the zona limitans intrathalamica exhibits differential activity on diencephalic molecular regionalization and nuclear structure. Neuroscience 143:129-140.

Vieira C, Garda AL, Shimamura K, Martinez S. 2005. Thalamic development induced by Shh in the chick embryo. Dev Biol 284:351-363.

Yoon MS, Puelles L, Redies C. 2000. Formation of cadherin-expressing brain nuclei in diencephalic alar plate divisions. J Comp Neurol 427: 461-480.

Zhou Q, Wang S, Anderson DJ. 2000. Identification of a novel family of oligodendrocyte lineage-specific basic helix-loop-helix transcription factors. Neuron 25:331-343.

Zhou CJ, Pinson KI, Pleasure SJ. 2004. Severe defects in dorsal thalamic development in low-density lipoprotein receptor-related protein-6 mutants. J Neurosci 24:7632-7639.

Zhuang B, Sockanathan S. 2006. Dorsal-ventral patterning: a view from the top. Curr Opin Neurobiol 16:20-24.

Zinyk DL, Mercer EH, Harris E, Anderson DJ, Joyner AL. 1998. Fate mapping of the mouse midbrain-hindbrain constriction using a sitespecific recombination system. Curr Biol 8:665-668. 